\title{
On Theorems of Hardy, Gelfand-Shilov and Beurling for Semisimple Groups
}

\author{
By \\ Sundaram Thangavelu* \\ For voices pursue him by day \\ And haunt him by night, \\ And he listens and needs must obey, \\ When the Angel says, "Write!"
}

- H. W. Longfellow

\begin{abstract}
In this paper we prove a strong version of Hardy's theorem for the group Fourier transform on semisimple Lie groups which characterises the Fourier transforms of all functions satisfying Hardy type conditions. In the particular case of $S L(2, \mathbb{R})$ we characterise all such functions and conjecture that the same is true for all rank one semisimple groups. We also establish an analogue of a theorem of Gelfand and Shilov in the context of semisimple groups. A version of Beurling's theorem which assumes a Cowling-Price condition on the function is also proved. We show that these results yield most of the earlier results as corollaries.
\end{abstract}

\section{$\S 1$. Introduction and the Main Results}

The aim of this paper is to establish a couple of uncertainty principles such as the theorems of Hardy, Gelfand-Shilov and Beurling for semisimple

Communicated by M. Kashiwara. Received November 14, 2002. Revised March 31, 2003. 2000 Mathematics Subject Classification(s): 22E30 (43A30)

Key words: Semisimple Lie groups, representations, Fourier transform, heat kernel, entire functions, symmetric spaces and Jacobi transform.

* Stat-Math Division, Indian Statistical Institute, 8th Mile, Mysore Road, Bangalore 560059 , India.

e-mail: veluma@isibang.ac.in 
Lie groups. First we revisit Hardy's theorem and prove a very general version of this theorem for the group Fourier transform. In the case of $S L(2, \mathbb{R})$ we find all the functions satisfying the Hardy conditions. Our second result is an analogue of a theorem of Gelfand and Shilov in the context of semisimple Lie groups. We also obtain a somewhat weak version of Beurling's theorem. Let us quickly set up the notations and state our main results. Details will be given in the following sections.

Let $G$ be a connected noncompact semisimple Lie group with finite centre. Let $K A N$ be an Iwasawa decomposition of $G$ and let $M A N$ be the associated minimal parabolic subgroup of $G$. As usual we denote the Lie algebras of $G$ and $A$ by $\mathfrak{g}$ and $\mathfrak{a}$ respectively. The norms on $\mathfrak{a}$ and $\mathfrak{a}^{*}$ induced by the Killing form are denoted by $|\cdot|$. For $\sigma \in \hat{M}$ and $\lambda \in \mathfrak{a}_{\mathbb{C}}^{*}$ let $\pi_{\lambda, \sigma}$ be the principal series representation associated with the minimal parabolic subgroup MAN. These are realised on a Hilbert space $H(K, \sigma)$. (In this paper we are only concerned with this principal series representations. As observed in [9], for a very rapidly decreasing function, the group Fourier transform being zero on all the principal series coming from the minimal parabolic subgroup necessarily implies that it is identically zero on the unitary dual.) If $x=k a k^{\prime}$ is the polar decomposition of $x \in G$ then we define $|x|=|\log a|$. For suitable functions $f$ on $G$ we define $\hat{f}(\lambda, \sigma)=\pi_{\lambda, \sigma}(f)$ for $\sigma \in \hat{M}$ and $\lambda \in \mathfrak{a}_{\mathbb{C}}^{*}$. We denote the operator norm of $\hat{f}(\lambda, \sigma)$ by $\|\hat{f}(\lambda, \sigma)\|$. With these notations we state our first theorem.

Theorem 1.1. Let $f \in L^{1}(G)$ satisfy the following two conditions for some $s>0, t>0$ with st $\geq \frac{1}{4}$.

(i) $|f(x)| \leq c(1+|x|)^{N} e^{-s|x|^{2}} e^{-\rho(\log a)}, x=k a k^{\prime} \in G$,

(ii) $\|\hat{f}(\lambda, \sigma)\| \leq c(1+|\lambda|)^{N} e^{-t|\lambda|^{2}}, \sigma \in \hat{M}, \lambda \in \mathfrak{a}^{*}$.

Then $\hat{f}(\lambda, \sigma)=0$ unless st $=\frac{1}{4}$ in which case

$$
\hat{f}(\lambda, \sigma)=e^{-t\left(\lambda_{1}^{2}+\cdots+\lambda_{\ell}^{2}\right)}\left(\sum_{|\alpha| \leq N} \lambda^{\alpha} A_{\alpha}\right)
$$

for all $\lambda \in \mathfrak{a}_{\mathbb{C}}^{*}$ and $\sigma \in \hat{M}$ where $A_{\alpha}$ are certain operators acting on $H(K, \sigma)$.

Actually, it turns out that $A_{\alpha}$ 's are the restrictions to $H(K, \sigma)$ of certain fixed operators which are given in terms of the function $f$. It is interesting to note that the conditions (i) and (ii) allow us to factorise $\hat{f}(\lambda, \sigma)$ as above with $A_{\alpha}$ independent of $\lambda$ and $\sigma$. We show that all the earlier versions of Hardy's theorem proved by Sitaram-Sundari [35], Cowling-Sitaram-Sundari [9], 
Sengupta [31], Narayanan-Ray [24] can be deduced from the above result. In particular we have the following result for right $K$-invariant functions on a semisimple group of rank one.

Let $G$ be such a group so that $G / K$ is a Riemannian symmetric space of rank one. Let $\Delta$ be the Laplace Beltrami operator on $G / K$ and let $p_{t}(x)$, $t>0$ stand for the heat kernel associated to $\Delta$. Then good estimates for $p_{t}(x)$ are known and we can replace the hypothesis (i) in Theorem 1.1 by the condition

$$
|f(x)| \leq C(1+|x|)^{m} p_{t}(x) .
$$

In what follows we denote by $\delta$ the class one representations of $K$ and by $Y_{\delta, j}$ the associated spherical harmonics on $K / M$. We also let $\Delta_{\delta}$ stand for certain Jacobi operators with associated heat kernels $p_{t}^{\delta}$. With these notations we have the following corollary.

Corollary 1.2. Let $f$ be a right $K$-invariant function on a rank one semisimple Lie group $G$. Assume that for some $t>0$, $f$ satisfies the conditions

(i) $|f(x)| \leq c(1+|x|)^{N} p_{t}(x), x \in G$,

(ii) $\|\hat{f}(\lambda, \sigma)\| \leq c(1+|\lambda|)^{N} e^{-t|\lambda|^{2}}, \sigma \in \hat{M}, \lambda \in \mathfrak{a}^{*}$.

Then $f\left(k a k^{\prime}\right)$ is a finite linear combination of functions of the form

$$
Y_{\delta, j}(k) P_{\delta}\left(\Delta_{\delta}\right) p_{t}^{\delta}(a)
$$

for some polynomials $P_{\delta}$ whose degrees do not exceed $N$.

The case $N=0$ is proved in Narayanan-Ray [24]; here we deduce the general case from Theorem 1.1. Observe that the equality case in Theorem 1.1 characterises the function $f$ only on the Fourier transform side. In general we are not able to say anything on the function itself. In the above Corollary we have restricted to the right $K$-invariant case and characterised functions satisfying the Hardy condition with $s=t$. When $N=0$ we have proved that $f(x)=c p_{t}(x)$. In the next theorem we consider the case $G=S L(2, \mathbb{R})$ and characterise all functions satisfying the Hardy conditions with $s=t$, thus proving a complete analogue of Hardy's theorem. Let us write the $K A K$ decomposition on $S L(2, \mathbb{R})$ as $x=k_{\theta} a_{r} k_{\varphi}$.

Theorem 1.3. Let $f$ be a function on $G=S L(2, \mathbb{R})$ which satisfies the conditions 
(i) $|f(x)| \leq c p_{t}(x), x \in G$,

(ii) $\|\hat{f}(\lambda, \sigma)\| \leq c e^{-t|\lambda|^{2}}, \sigma \in \hat{M}, \lambda \in \mathfrak{a}^{*}$.

Then

$$
f\left(k_{\theta} a_{r} k_{\varphi}\right)=\sum_{m=-\infty}^{\infty}\left(e^{-i m(\theta+\varphi)} f_{m}(r)+e^{-i\left(m+\frac{1}{2}\right)(\theta+\varphi)} f_{m+\frac{1}{2}}(r)\right)
$$

where $f_{m}$ and $f_{m+\frac{1}{2}}$, defined in terms of heat kernels associated to certain Jacobi operators, satisfy the same estimate as $p_{t}\left(a_{r}\right)$.

Thus we see that in the equality case in Theorem 1.1 (for $G=S L(2, \mathbb{R})$ ) there are infinitely many linearly independent functions satisfying the hypotheses of the theorem. We conjecture that the same is true for all rank one semisimple Lie groups. Compare the above result with the corresponding result in Sarkar-Thangavelu [30] for the Euclidean motion group.

Our next theorem is an analogue of a theorem of Gelfand-Shilov which was originally proved for the Fourier transform on $\mathbb{R}$. Recently Bonami et al. [6] have established a general version of this theorem for the Fourier transform on $\mathbb{R}^{n}$. Here we establish the following result for semisimple Lie groups.

Theorem 1.4. Let $f \in L^{1}(G)$ satisfy the following two conditions:

(i) $\int_{G}|f(x)|(1+|x|)^{-N} e^{\frac{a^{p}}{p}|x|^{p}} d x<\infty$,

(ii) $\int_{\mathfrak{a}^{*}}\|\hat{f}(\lambda, \sigma)\|(1+|\lambda|)^{-N} e^{\frac{b^{q}}{q}|\lambda|^{q}} d \lambda<\infty$

for every $\sigma \in \hat{M}$ where $N \geq 0, a b \geq 1,1<q \leq 2$ and $\frac{1}{p}+\frac{1}{q}=1$. Then $f=0$ unless $p=q=2$ and $a b=1$ in which case $\hat{f}(\lambda, \sigma)$ can be represented as

$$
\hat{f}(\lambda, \sigma)=e^{-\left(E_{\sigma} \lambda, \lambda\right)}\left(\sum_{|\alpha| \leq N} \lambda^{\alpha} A_{\alpha}\right)
$$

where $A_{\alpha}$ are operators on $H(K, \sigma)$ and $E_{\sigma}$ is symmetric with $R e E_{\sigma} \geq \frac{1}{2} b^{2} I$.

The Euclidean analogues of Theorems 1.1 and 1.2 can be deduced from a much stronger result known as Beurling's theorem which was established in Bonami et al. [6] in the most general form. This result says that if a function $f$ on $\mathbb{R}^{n}$ and its Fourier transform $\hat{f}$ satisfy the integrability condition

$$
\int_{\mathbb{R}^{n}} \int_{\mathbb{R}^{n}}|f(x)||\hat{f}(y)|(1+|x|+|y|)^{-N} e^{|(x, y)|} d x d y<\infty
$$


then $f$ can be written as $f(x)=P(x) e^{-\frac{1}{2}(A x, x)}$ where $P$ is a polynomial of degree $\leq N-n$ and $A$ is a positive definite matrix. Note the perfect symmetry of the above condition in $f$ and $\hat{f}$. The lack of such symmetry is a serious obstacle in establishing an analogue for semisimple groups.

We conjecture that a condition of the form

$$
\int_{G} \int_{\mathfrak{a}^{*}}|f(x)|\|\hat{f}(\lambda, \sigma)\|(1+|\lambda|)^{-N}(1+|x|)^{-N} e^{|\lambda||x|} d \lambda d x<\infty
$$

should lead to the conclusion that

$$
\hat{f}(\lambda, \sigma)=e^{-\left(E_{\sigma} \lambda, \lambda\right)}\left(\sum_{|\alpha| \leq N} \lambda^{\alpha} A_{\sigma, \alpha}\right)
$$

where $R e E_{\sigma} \geq 0$. In the Euclidean case if $f$ satisfies (1.1) then it is possible to find another function $g$ satisfying the same condition but with the added property that $\hat{g}(y)$ extends to $\mathbb{C}^{n}$ as an entire function of order 2 . This fact which is very crucial in the proof of Beurling's theorem seems to be very difficult to establish in the case of semisimple groups under the only hypothesis (1.2). However, once we assume this fact, a version of Beurling's theorem can be proved.

Theorem 1.5. Let $f \in L^{1}(G)$ satisfy the condition, for some $N \geq 0$,

$$
\int_{G} \int_{\mathfrak{a}^{*}} \frac{|f(x)|}{(1+|x|)^{N}} \frac{\|\hat{f}(\lambda, \sigma)\|}{(1+|\lambda|)^{N}} e^{|\lambda||x|} d \lambda d x<\infty
$$

for every $\sigma \in \hat{M}$. Further assume that the function $f$ satisfies

$$
\int_{G}|f(x)|^{p} e^{a p|x|^{2}} d x<\infty
$$

for some $a>0$ and $1 \leq p<\infty$. Then $\hat{f}(\lambda, \sigma)$ can be written as in (1.3).

For the Euclidean Fourier transform there is one more uncertainty principle due to Cowling and Price [8]. This says that if $f$ and $\hat{f}$ satisfy the conditions

$$
\int_{\mathbb{R}^{n}}|f(x)|^{p} e^{a p|x|^{2}} d x<\infty, \quad \int_{\mathbb{R}^{n}}|\hat{f}(y)|^{q} e^{b q|y|^{2}} d y<\infty
$$

for some $1 \leq p, q \leq \infty$ and $a b>\frac{1}{4}$ then $f=0$. A general form of this theorem also follows from Beurling's theorem as shown in [6]. Our corollary above combines Beurling's condition with a condition of Cowling-Price 
type. (For this reason my friend Rudra named the above result as Cowbeurling theorem!).

We conclude this introduction with some historical remarks. In 1933, Hardy [16] established the first 'Hardy's theorem' for the Fourier transform on $\mathbb{R}$. Extensions of this theorem to $\mathbb{R}^{n}$ and refinements were obtained in Sitaram et al. [34], Pfannschmidt [25] and Thangavelu [39]. The first version of Hardy's theorem for semisimple Lie groups with one conjugacy class of Cartan subgroups was proved in Sitaram-Sundari [35]. Later this condition was removed by Cowling et al. in [9]. Another proof was given by Sengupta [31]. Narayanan-Ray [24] considered the equality case of Hardy's theorem in the context of symmetric spaces. In [39] we obtained a refined version of Hardy's theorem for rank one symmetric spaces. Some $L^{p}$ versions were proved by Narayanan-Ray [23] and Sarkar-Ray [27]. Sarkar [28] and [29] have treated the equality case of Hardy's theorem for the full group but the results obtained are at the level of matrix coefficients.

The story of Hardy's theorem for Heisenberg and other nilpotent groups can be read from the papers of the author [37], [38], those of Ray [26], Astengo et al. [4] and Kaniuth-Kumar [19]. Recently the author has proved a version of Beurling's theorem for step 2 nilpotent groups. In [30] Sarkar-Thangavelu have established the most general Hardy's theorem for the Euclidean motion group; they also have proved a version of Beurling's theorem. For earlier works on motion groups we refer to Sundari [36] and Eguchi et al. [10], [11]. The results in this paper completely characterise the functions satisfying Hardy conditions on the Fourier transform side. But only in some special cases such as rank one symmetric spaces we are able to say something about the function itself. So, still there is room for improvement of the results presented here.

We wish to thank Rudra Sarkar for answering many naive questions on the representation theory of semisimple groups. It is also my pleasure to thank Ms. Asha Lata who transformed my notes into a respectable manuscript. We also wish to thank the referee for his careful reading of the manuscript and also for pointing out the reference [33].

\section{$\S 2 . \quad$ Preliminaries}

Let $G$ be a connected noncompact semisimple Lie group with finite centre and fix an Iwasawa decomposition $G=K A N$. Here $K$ is maximal compact, $A$ is abelian and $N$ is nilpotent. Let $\mathfrak{a}$ be the Lie algebra of $A$ which will be identified with $\mathbb{R}^{\ell}$ under the inner product defined by the Cartan-Killing form on the Lie algebra $\mathfrak{g}$ of $G$. We then fix a Weyl chamber $\mathfrak{a}^{+}$and with 
$A^{+}=\exp \mathfrak{a}^{+}$obtain the polar decomposition $G=K \bar{A}^{+} K$. Thus each $x \in G$ can be written as $x=k_{1} a k_{2}$ with $k_{1}, k_{2} \in K$ and $a \in \bar{A}^{+}$. We then define $|x|=|\log a|$, where $\log a$ stands for the element in $\mathfrak{a}^{+}$for which $\exp (\log a)=a$. Let $\Lambda$ be the set of positive roots corresponding to the Weyl chamber $\mathfrak{a}^{+}$. For each $\alpha \in \Lambda$ let $\mathfrak{g}_{\alpha}$ be the root space corresponding to $\alpha$. Define the function $J(a)$ by

$$
J(a)=\prod_{\alpha \in \Lambda}\left(e^{\alpha(\log a)}-e^{-\alpha(\log a)}\right)^{n(\alpha)}
$$

where $n(\alpha)$ is the dimension of $\mathfrak{g}_{\alpha}$. Then the Haar measure $d x$ on $G$ takes the form $d x=J(a) d a d k_{1} d k_{2}$ in terms of the polar decomposition $x=k_{1} a k_{2}$. The function $J(a)$ satisfies the estimate

$$
|J(a)| \leq c e^{2 \rho(\log a)}
$$

where $\rho$ is the element of $\mathfrak{a}^{*}$ defined by

$$
\rho(\log a)=\frac{1}{2} \sum_{\alpha \in \Lambda} n(\alpha) \alpha(\log a) .
$$

We now describe the principal series representations of $G$. Let $M$ be the centraliser of $A$ in $K$ so that $P=M A N$ is the minimal parabolic subgroup of $G$. The Iwasawa decomposition gives rise to the mappings $a: G \rightarrow A, \kappa: G \rightarrow K$ and $n: G \rightarrow N$ so that

$$
x=\kappa(x) a(x) n(x)=k(x) \exp H(x) n(x)
$$

where $H(x)=\log a(x) \in \mathfrak{a}$. The function $H\left(x^{-1} k\right)$ is then right $K$-invariant in $x$ and right $M$-invariant in $K$. For every $\sigma \in \hat{M}$ let $H_{\sigma}$ be the finite dimensional Hilbert space on which $\sigma$ is realised. If $d_{\sigma}=\operatorname{dim} H_{\sigma}$ then we can identify $H_{\sigma}$ with $\mathbb{C}^{d_{\sigma}}$. Let $H(K, \sigma)$ stand for the Hilbert space of functions $\varphi: K \rightarrow \mathbb{C}^{d_{\sigma}}$ which satisfy the condition

$$
\varphi(k m)=\sigma(m)^{*} \varphi(k), k \in K, m \in M
$$

and for which

$$
\|\varphi\|_{2}^{2}=\int_{K}\|\varphi(k)\|_{H_{\sigma}}^{2} d k<\infty .
$$

This space is equipped with the inner product

$$
(\varphi, \psi)=\int_{K}(\varphi(k), \psi(k)) d k .
$$


For each $\lambda \in \mathfrak{a}_{\mathbb{C}}^{*}$ we define a representation $\pi_{\lambda, \sigma}$ on this Hilbert space by

$$
\pi_{\lambda, \sigma}(x) \varphi(k)=e^{-(i \lambda+\rho)\left(H\left(x^{-1} k\right)\right)} \varphi\left(\kappa\left(x^{-1} k\right)\right) .
$$

Then it is well known that $\pi_{\lambda, \sigma}$ is a unitary representation of $G$ whenever $\lambda \in \mathfrak{a}^{*}$. It is also known that $\pi_{\lambda, \sigma}$ and $\pi_{\mu, \sigma}$ are unitarily equivalent if and only if $\lambda=s \mu$ where $s$ belongs to the Weyl group. We refer to Varadarajan [40] and Knapp [20] for more about the representation theory of semisimple Lie groups.

For $f \in L^{1}(G)$ the group Fourier transform of $f$ is the operator valued function

$$
\hat{f}(\lambda, \sigma)=\pi_{\lambda, \sigma}(f)=\int_{G} f(x) \pi_{\lambda, \sigma}(x) d x
$$

for all $\lambda \in \mathfrak{a}^{*}$ and $\sigma \in \hat{M}$. Note that $\hat{f}(\lambda, \sigma)$ is a bounded linear operator on $H(K, \sigma)$ whose operator norm satisfies $\|\hat{f}(\lambda, \sigma)\| \leq\|f\|_{1}$. If the function $f$ has enough decay we can even define $\hat{f}(\lambda, \sigma)$ for $\lambda \in \mathfrak{a}_{\mathbb{C}}^{*}$ by the formula

$$
(\hat{f}(\lambda, \sigma) \varphi, \psi)=\int_{G} f(x)\left(\pi_{\lambda, \sigma}(x) \varphi, \psi\right) d x
$$

for $\varphi, \psi \in H(K, \sigma)$. The matrix coefficients $\left(\pi_{\lambda, \sigma}(x) \varphi, \psi\right)$ can be estimated using properties of the $H$ function. We record this estimate in the following.

Lemma 2.1. For every $\lambda \in \mathfrak{a}_{\mathbb{C}}^{*}$ and $\varphi, \psi \in H(K, \sigma)$ we have the estimate

$$
\left|\left(\pi_{\lambda, \sigma}(x) \varphi, \psi\right)\right| \leq\|\varphi\|_{2}\|\psi\|_{2} e^{|\operatorname{Im}(\lambda)||x|} .
$$

Proof. We need to estimate

$$
\left\|\pi_{\lambda, \sigma}(x) \varphi\right\|_{2}^{2}=\int_{K}\left\|e^{-(i \lambda+\rho)\left(H\left(x^{-1} k\right)\right)} \varphi\left(\kappa\left(x^{-1} k\right)\right)\right\|_{H_{\sigma}}^{2} d k .
$$

We identify $\lambda \in \mathfrak{a}_{\mathbb{C}}^{*}$ with an element of $\mathbb{C}^{\ell}$ where $\ell$ is the rank of $G$. Writing $\lambda=\lambda_{R}+i \lambda_{I}$ where $\lambda_{I}=\operatorname{Im}(\lambda)$ we see that

$$
\left\|\pi_{\lambda, \sigma}(x) \varphi\right\|_{2}^{2}=\int_{K} e^{2 \lambda_{I}\left(H\left(x^{-1} k\right)\right)} e^{-2 \rho\left(H\left(x^{-1} k\right)\right)}\left\|\varphi\left(\kappa\left(x^{-1} k\right)\right)\right\|_{H_{\sigma}}^{2} d k .
$$

If we write $x=k_{1} a k_{2}$ then as $H\left(x^{-1} k\right)$ is right $K$-invariant, we have $H\left(x^{-1} k\right)=$ $H\left(a^{-1} k_{1}^{-1} k\right)$. We can assume that $\lambda_{I}$ is the dominant vector in the orbit of $\lambda_{I}$ under the Weyl group. Then Corollary 3.5.3 in Gangolli-Varadarajan [15] shows that

$$
\lambda_{I}\left(H\left(x^{-1} k\right)\right)=\lambda_{I}\left(H\left(a^{-1} k_{1}^{-1} k\right)\right) \leq \lambda_{I}(\log a) .
$$


Therefore,

$$
\left\|\pi_{\lambda, \sigma}(x) \varphi\right\|_{2}^{2} \leq e^{2|\operatorname{Im}(\lambda)||x|} \int_{K} e^{-2 \rho\left(H\left(x^{-1} k\right)\right)}\left\|\varphi\left(\kappa\left(x^{-1} k\right)\right)\right\|_{H_{\sigma}}^{2} d k .
$$

Since the last integral is nothing but $\|\varphi\|_{2}^{2}$ we obtain the estimate.

We remark that earlier authors on Hardy's theorem (e.g. [9] and [35]) have used the estimate

$$
\left|\left(\pi_{\lambda, \sigma}(x) \varphi, \psi\right)\right| \leq C_{\varphi, \psi}(1+|x|)^{m} e^{|\operatorname{Im}(\lambda)||x|} e^{-\rho(\log a)}
$$

where the constant $C_{\varphi, \psi}$ depends on the $L^{\infty}$ norms of $\varphi$ and $\psi$ rather than their $L^{2}$ norms. The more precise estimates of Lemma 2.1 are required to prove the general version of Hardy's theorem.

In the above we have described only the principal series representations $\pi_{\lambda, \sigma}$. There are groups without discrete series representations for which the Plancherel measure is supported on the principal series itself. For example, if we assume that $G$ has only one conjugacy class of Cartan subgroups then there is an explicit measure $\mu(\sigma, \lambda)=\mu_{\sigma}(\lambda)$ on $\hat{M} \times \mathfrak{a}^{*}$ such that the following Plancherel formula holds: for every $f \in L^{1} \cap L^{2}(G)$,

$$
\int_{G}|f(x)|^{2} d x=\sum_{\sigma} \int_{\mathfrak{a}^{*}}\|\hat{f}(\lambda, \sigma)\|_{H S}^{2} d \mu_{\sigma}(\lambda) .
$$

Here and elsewhere $\|\hat{f}(\lambda, \sigma)\|_{H S}$ stands for the Hilbert-Schmidt operator norm of $\hat{f}(\lambda, \sigma)$. For a suitable class of functions we also have the inversion formula

$$
f(x)=\sum_{\sigma \in \hat{M}} \int_{\mathfrak{a}^{*}} \operatorname{tr}\left(\hat{f}(\lambda, \sigma) \pi_{\lambda, \sigma}(x)^{*}\right) d \mu_{\sigma}(\lambda) .
$$

The inversion and Plancherel formulas become more complicated in the presence of discrete series representations.

There is a convenient way of realising the Fourier transform $\hat{f}$ on a fixed Hilbert space. This can be achieved by using the generalised Fourier transform studied by Gross-Kunze [14]. Let $L^{2}(K, \sigma)$ be the direct sum of $d_{\sigma}$ copies of $H(K, \sigma)$ and define $L^{2}(K)^{\wedge}$ as the direct sum of the Hilbert spaces $L^{2}(K, \sigma)$. Then there is a unitary operator $U: L^{2}(K) \rightarrow L^{2}(K)^{\wedge}$ which has the property that $U^{*} L^{2}(K, \sigma)$, where $U^{*}: L^{2}(K)^{\wedge} \rightarrow L^{2}(K)$ is the formal adjoint of $U$, is precisely the Hilbert subspace of functions $\psi \in L^{2}(K)$ satisfying the equation

$$
\psi(k)=\int_{M} \psi\left(u^{-1} k\right) \sigma(u) d u .
$$


We can think of the group Fourier transform as an operator valued function on $\mathfrak{a}^{*}$ given by

$$
\hat{f}(\lambda) \varphi=\sum_{\sigma \in \hat{M}} U^{*} \hat{f}(\lambda, \sigma) U \varphi_{\sigma}
$$

whenever $\varphi \in L^{2}(K)$ has the representation

$$
\varphi=\sum_{\sigma \in \hat{M}} \varphi_{\sigma}, \quad \varphi_{\sigma} \in U^{*} L^{2}(K, \sigma) .
$$

Thus for $\lambda \in \mathfrak{a}^{*}, \hat{f}(\lambda)$ is a bounded linear operator on $L^{2}(K)$. The conclusions of Theorem 1.1 and Corollary 1.2 then takes the form

$$
\hat{f}(\lambda)=e^{-t \lambda^{2}}\left(\sum_{|\alpha| \leq N} \lambda^{\alpha} A_{\alpha}\right)
$$

where $\lambda^{2}=\lambda_{1}^{2}+\cdots+\lambda_{\ell}^{2}$ and $A_{\alpha}$ are certain operators acting on $L^{2}(K)$.

\section{$\S 3 . \quad H a r d y ' s$ Theorem for Semisimple Groups}

We now give a proof of Theorem 1.1 and show how to deduce earlier versions as corollaries. As in the case of previous versions of Hardy's theorem, we require some complex analytic lemmas dealing with entire functions of order 2. The following lemma, which can be proved using the Phragman-Lindelöf maximum principle, is one such result which we require.

Lemma 3.1. Let $F(z)$ be an entire function of $\ell$ complex variables $z=$ $\left(z_{1}, z_{2}, \ldots, z_{\ell}\right)$ which satisfies the estimates

$$
\begin{aligned}
& |F(z)| \leq c(1+|z|)^{m} e^{a|\operatorname{Im}(z)|^{2}}, z \in \mathbb{C}^{\ell} \\
& |F(x)| \leq c(1+|x|)^{m} e^{-a|x|^{2}}, x \in \mathbb{R}^{\ell}
\end{aligned}
$$

for a constant $a>0$. Then $F(z)=P(z) e^{-a z^{2}}$ where $P(z)$ is a polynomial of degree $\leq m$ and $z^{2}$ stands for $z_{1}^{2}+z_{2}^{2}+\cdots+z_{\ell}^{2}$.

A proof of this lemma can be found in Sarkar [28] and Narayanan-Ray [23]. The version $m=0, \ell=1$ was used by Hardy and the case $m=0, \ell>1$ was proved and used by Sitaram-Sundari [35]. We now turn to a proof of Theorem 1.1.

We first consider the case $s t=\frac{1}{4}$ of Theorem 1.1. Let us fix an orthonormal basis for $H(K, \sigma)$ consisting of $K$-finite vectors. Let $\varphi$ and $\psi$ be any two 
members of this basis and consider the function $F(\lambda)=(\hat{f}(\lambda, \sigma) \varphi, \psi)$. We claim that $F(\lambda)$ extends to $\lambda \in \mathfrak{a}_{\mathbb{C}}^{*}$ as an entire function of order 2 . To see this we have

$$
F(\lambda)=\int_{G} f(x)\left(\pi_{\lambda, \sigma}(x) \varphi, \psi\right) d x
$$

We are given that

$$
\left|f\left(k a k^{\prime}\right)\right| \leq c(1+|\log a|)^{N} e^{-s|\log a|^{2}} e^{-\rho(\log a)}
$$

and by Lemma 2.1 we also have

$$
\left|\left(\pi_{\lambda, \sigma}\left(k a k^{\prime}\right) \varphi, \psi\right)\right| \leq c\|\varphi\|_{2}\|\psi\|_{2} e^{|\log a||\operatorname{Im}(\lambda)|} .
$$

Using these two estimates in (3.1) we have

$$
|F(\lambda)| \leq c\|\varphi\|_{2}\|\psi\|_{2} \int_{A}(1+|\log a|)^{N} e^{-s|\log a|^{2}} e^{\rho(\log a)} e^{|\log a||\operatorname{Im}(\lambda)|} d a
$$

where we have made use of the estimate

$$
|J(a)| \leq c e^{2 \rho(\log a)} .
$$

The above leads to the estimate

$$
|F(\lambda)| \leq c(1+|\lambda|)^{m} e^{t(|\operatorname{Im}(\lambda)|+|\rho|)^{2}}\|\varphi\|_{2}\|\psi\|_{2}
$$

as $s t=\frac{1}{4}$. Here $m$ depends on $N$ and $\ell$.

This shows that $F(\lambda)$ extends to $\mathfrak{a}_{\mathbb{C}}^{*}$ as an entire function of order 2 which satisfies (3.4). Instead of Lemma 2.1 we can also use the slightly stronger estimate (2.5). This will lead to the estimate

$$
|F(\lambda)| \leq C_{\varphi, \psi}(1+|\lambda|)^{m} e^{t|\operatorname{Im}(\lambda)|^{2}}, \lambda \in \mathfrak{a}_{\mathbb{C}}^{*}
$$

Here the constant $C_{\varphi, \psi}$ depends on $\|\varphi\|_{\infty}$ and $\|\psi\|_{\infty}$. The hypothesis on $\hat{f}(\lambda, \sigma)$ gives us

$$
|F(\lambda)| \leq C\|\varphi\|_{2}\|\psi\|_{2}(1+|\lambda|)^{N} e^{-t|\lambda|^{2}}, \lambda \in \mathfrak{a}^{*} .
$$

In view of (3.5) and (3.6) we are in a position to apply Lemma 3.1 to conclude that

$$
(\hat{f}(\lambda, \sigma) \varphi, \psi)=e^{-t \lambda^{2}} P_{\sigma}(\lambda, \varphi, \psi)
$$


where $P_{\sigma}(\lambda, \varphi, \psi)$ is a polynomial of degree $\leq N$. Here we have written $\lambda^{2}$ to stand for $\lambda_{1}^{2}+\cdots+\lambda_{\ell}^{2}$. From (3.7) it is clear that $P_{\sigma}(\lambda, \varphi, \psi)$ is linear in $\varphi$ and $\psi$. Writing

$$
P_{\sigma}(\lambda, \varphi, \psi)=\sum_{|\alpha| \leq N} C_{\sigma, \alpha}(\varphi, \psi) \lambda^{\alpha}
$$

we can easily check that the coefficients $C_{\sigma, \alpha}(\varphi, \psi)$ are sesquilinear functionals on $H(K, \sigma)$. Now we can use the estimate (3.4) together with Cauchy's integral formula to show that $C_{\sigma, \alpha}(\varphi, \psi)$ are bounded: that is, they satisfy estimates

$$
\left|C_{\sigma, \alpha}(\varphi, \psi)\right| \leq C_{\sigma, \alpha}\|\varphi\|_{2}\|\psi\|_{2}
$$

We have proved the above estimates for all $\varphi$ and $\psi$ coming from an orthonormal basis. Clearly the same estimate is valid for all $\varphi$ and $\psi$ belonging $H(K, \sigma)$. Therefore, there exist bounded linear operators $A_{\sigma, \alpha}$ on $H(K, \sigma)$ such that $C_{\sigma, \alpha}(\varphi, \psi)=\left(A_{\sigma, \alpha} \varphi, \psi\right)$. Thus we have the equation

$$
\hat{f}(\lambda, \sigma)=e^{-t \lambda^{2}}\left(\sum_{|\alpha| \leq N} \lambda^{\alpha} A_{\sigma, \alpha}\right) .
$$

Now from the definition of $\hat{f}(\lambda, \sigma)$ we have

$$
\hat{f}(\lambda, \sigma) \varphi(k)=\int_{G} f(x) e^{-(i \lambda+\rho)\left(H\left(x^{-1} k\right)\right)} \varphi\left(\kappa\left(x^{-1} k\right)\right) d x .
$$

We can calculate $A_{\sigma, \alpha} \varphi(k)$ by taking derivatives of $e^{t \lambda^{2}} \hat{f}(\lambda, \sigma) \varphi(k)$ with respect to $\lambda$ at $\lambda=0$. In fact, it is easily seen that $A_{\sigma, \alpha} \varphi(k)$ is a finite linear combination of operators of the form

$$
\int_{G} f(x) e^{-\rho\left(H\left(x^{-1} k\right)\right)}\left(H\left(x^{-1} k\right)\right)^{\beta} \varphi\left(\kappa\left(x^{-1} k\right)\right) d x .
$$

Thus there exist fixed operators $A_{\alpha}$ whose restriction to $H(K, \sigma)$ coincides with $A_{\sigma, \alpha}$. Hence

$$
\hat{f}(\lambda, \sigma)=e^{-t \lambda^{2}}\left(\sum_{|\alpha| \leq N} \lambda^{\alpha} A_{\alpha}\right)
$$

and this proves the case $s t=\frac{1}{4}$ of Theorem 1.1.

When st $>\frac{1}{4}$, it is clear that $\hat{f}(\lambda, \sigma)$ satisfies $\|\hat{f}(\lambda, \sigma)\| \leq c(1+|\lambda|)^{N}$ $e^{-\frac{1}{4 s}|\lambda|^{2}}$. Hence we conclude that

$$
\hat{f}(\lambda, \sigma)=e^{-\frac{1}{4 s} \lambda^{2}}\left(\sum_{|\alpha| \leq N} \lambda^{\alpha} A_{\alpha}\right) .
$$


But then the estimate

$$
\|\hat{f}(\lambda, \sigma)\| \leq c(1+|\lambda|)^{N} e^{-t|\lambda|^{2}}
$$

forces all $A_{\alpha}$ to be zero. Hence $\hat{f}(\lambda, \sigma)=0$ for every $\lambda \in \mathfrak{a}^{*}$ and $\sigma \in \hat{M}$.

As corollaries of Theorem 1.1 we have the following results.

Corollary 3.2 (Sitaram-Sundari). Assume that $G$ has only one conjugacy class of Cartan subgroups. If $f$ satisfies

$$
|f(x)| \leq c e^{-\alpha|x|^{2}},\|\hat{f}(\lambda, \sigma)\|_{H S} \leq c e^{-\beta|\lambda|^{2}}
$$

for all $x \in G, \lambda \in \mathfrak{a}^{*}$ and $\sigma \in \hat{M}$ then $f=0$ whenever $\alpha \beta>\frac{1}{4}$.

For such groups the Plancherel measure is supported on $\hat{M} \times \mathfrak{a}^{*}$ and hence we only need to show that $\hat{f}(\lambda, \sigma)=0$ for all $\lambda \in \mathfrak{a}^{*}, \sigma \in \hat{M}$. As $f$ and $\hat{f}$ clearly satisfy the conditions of Theorem 1.1, Corollary 3.2 follows immediately. As shown in Cowling et al. [9] the condition on $G$ can be removed by using HarishChandra's subquotient theorem. We refer to [9] for details.

The equality case $\left(s t=\frac{1}{4}\right)$ of Hardy's theorem for semisimple groups was considered by Narayanan-Ray [24] for right $K$-invariant functions. Here is their theorem. A slightly weaker form of the result is also known to Shimeno [33].

Corollary 3.3 (Narayanan-Ray). Let $f$ be a right $K$-invariant function on $G$ which satisfies the estimates

$$
\begin{aligned}
|f(x)| & \leq c(1+|x|)^{N} e^{-\frac{1}{4 t}|x|^{2}} e^{-\rho(\log a)}, x \in G \\
\|\hat{f}(\lambda, 1)\| & \leq c e^{-t|\lambda|^{2}}, \lambda \in \mathfrak{a}^{*} .
\end{aligned}
$$

Then $f$ is a constant multiple of the heat kernel $p_{t}$ associated to the LaplaceBeltrami operator on $G / K$.

We pause for a moment to say few words about the heat kernel $p_{t}(x)$. It is well known that the solution of the heat equation

$$
\frac{\partial}{\partial t} u(x, t)=\Delta u(x, t), u(x, 0)=f(x)
$$

is given by the convolution $u(x, t)=f * p_{t}(x)$ with a $K$-biinvariant function $p_{t}(x)$. This function is called the heat kernel and plays the role of Gaussian on the symmetric space $G / K$. As $p_{t}$ is $K$-biinvariant, $\hat{p}_{t}(\lambda, \sigma)=0$ for all $\sigma$ other than $\sigma=1$ and

$$
\hat{p}_{t}(\lambda, 1)=e^{-t\left(|\lambda|^{2}+|\rho|^{2}\right)} A
$$


where the operator $A: L^{2}(K / M) \rightarrow L^{2}(K / M)$ has a very simple description. There is an orthonormal basis $\left\{v_{j}: j \geq 0\right\}$ with $v_{0}(k)=1$ for $L^{2}(K / M)$ such that $A v_{0}=v_{0}$ and $A v_{j}=0$ for $j \geq 1$. Thus, $\hat{p}_{t}(\lambda, 1)$ can be identified with the scalar $\hat{p}_{t}(\lambda)=\left(\hat{p}_{t}(\lambda, 1) v_{0}, v_{0}\right)$ which equals $e^{-t\left(|\lambda|^{2}+|\rho|^{2}\right)}$.

Returning to Corollary 3.3, let $f$ be a right $K$-invariant function on $G$. Then $\hat{f}(\lambda, \sigma)=0$ for all $\sigma \neq 1$. When $\sigma=1, \pi_{\lambda, \sigma}$ are called spherical principal series representations, realised on $L^{2}(K / M)$. Writing $\hat{f}(\lambda)$ in place of $\hat{f}(\lambda, 1)$, we obtain from Theorem 1.1 that $\hat{f}(\lambda)=e^{-t|\lambda|^{2}} A$. Then

$$
\left(A v_{0}, v_{j}\right)=e^{t|\lambda|^{2}} \int_{G} f(x)\left(\pi_{\lambda}(x) v_{0}, v_{j}\right) d x .
$$

Since $v_{0}=1$ the above integral is equal to

$$
\int_{G} \int_{K / M} f(x) e^{-(i \lambda+\rho)\left(H\left(x^{-1} k\right)\right)} \bar{v}_{j}(k) d k d x .
$$

But the inner integral vanishes at $\lambda=i \rho$ for all $j \neq 0$. Hence $A v_{0}=c v_{0}$; the right $K$-invariance of $f$ already implies $A v_{j}=0$ for $j \neq 0$. Hence it follows that $f$ is a constant multiple of the heat kernel.

Using Theorem 1.1 we can prove a stronger version of Corollary 3.3. Before doing that we collect some more information regarding heat kernels on Riemannian symmetric spaces. There is a conjecture due to Anker [3] which says that the heat kernel $p_{t}(x)$ associated to the Laplace-Beltrami operator $\Delta$ on $G / K$ satisfies the estimate

$$
p_{t}(x) \leq c_{t} P(|\log a|) e^{-|\rho|^{2} t} e^{-\frac{1}{4 t}|\log a|^{2}} e^{-\rho(\log a)}
$$

where $P$ is an explicit polynomial defined in terms of indivisible positive roots. The conjecture has been proved for all complex semisimple groups and also for groups of real rank one. The estimates are also known for $S L(n, \mathbb{R})$ and $S U(p, q)$. We refer to the works of Anker [1] and Anker-Ji [3].

For all groups $G$ for which the above estimate is known, we can replace the hypothesis in Theorem 1.1 by

$$
|f(x)| \leq c(1+|x|)^{N} p_{s}(x),\|\hat{f}(\lambda, \sigma)\| \leq c(1+|\lambda|)^{N} e^{-t|\lambda|^{2}}
$$

and the conclusion is: $\hat{f}(\lambda, \sigma)=0$ for $s<t$ and

$$
\hat{f}(\lambda, \sigma)=e^{-t \lambda^{2}}\left(\sum_{|\alpha| \leq N} \lambda^{\alpha} A_{\alpha}\right)
$$


when $s=t$. In the case of right $K$-invariant functions when $N=0$ and $s=t$ we arrive at the conclusion that $f(x)=c p_{t}(x)$. Thus the equality case in Hardy's theorem can be viewed as a result characterising heat kernels.

When we leave the premises of right $K$-invariant functions then the conditions (3.15) imply that $\hat{f}(\lambda, \sigma)=e^{-t|\lambda|^{2}} A$ but from this we cannot conclude that $f$ is a constant multiple of the heat kernel. However, we have the following.

Corollary 3.4. Let $G$ be a group for which (3.14) is known and let $f$ be a function on $G$ which satisfies (3.15) with $N=0, s=t$. Then

$$
\int_{K} f(x k) d k=c p_{t}(x)
$$

Proof. For any $\epsilon>0$ the function $f * p_{\epsilon}(x)=f_{\epsilon}(x)$ is right $K$-invariant and satisfies

$$
\left|f_{\epsilon}(x)\right| \leq c p_{t} * p_{\epsilon}(x)=c p_{t+\epsilon}(x)
$$

and also the condition

$$
\|\hat{f}(\lambda, \sigma)\| \leq c e^{-(t+\epsilon)|\lambda|^{2}} .
$$

Hence, $f * p_{\epsilon}(x)=c p_{t+\epsilon}(x)$. Taking limit as $\epsilon \rightarrow 0$ and noting that

$$
\lim _{\epsilon \rightarrow 0} f * p_{\epsilon}(x)=\int_{K} f(x k) d k
$$

we complete the proof of the corollary.

Let us assume that the group $G$ is such that the Plancherel measure is supported on the principal series itself. Then the conclusion

$$
\hat{f}(\lambda, \sigma)=e^{-t|\lambda|^{2}} A
$$

along with the inversion formula leads to

$$
f(x)=\sum_{\sigma \in \hat{M}} \int_{\mathfrak{a}^{*}} e^{-t|\lambda|^{2}} \operatorname{tr}\left(A \pi_{\lambda, \sigma}(x)^{*}\right) d \mu_{\sigma}(\lambda) .
$$

Consider the Casimir operator $\Omega$ which is in the centre of the universal enveloping algebra of $\mathfrak{g}$. As such $\pi_{\lambda, \sigma}(\Omega)$ is a scalar which can be explicitly calculated for certain groups and we have $\pi_{\lambda, \sigma}(\Omega)=-|\lambda|^{2}-c_{\sigma}^{2}$ for some constants $c_{\sigma}$. Defining

$$
f_{\sigma}(x, t)=\int_{\mathfrak{a}^{*}} e^{-t|\lambda|^{2}} \operatorname{tr}\left(A \pi_{\lambda, \sigma}(x)^{*}\right) d \mu_{\sigma}(\lambda)
$$


we can write

$$
f(x)=\sum_{\sigma \in \hat{M}} f_{\sigma}(x, t) .
$$

The functions $f_{\sigma}$ can be called generalised heat kernels for the following reason.

For $\varphi$ and $\psi$ in $H(K, \sigma)$ it is clear that for any left invariant vector field $X$

$$
X\left(\pi_{\lambda, \sigma}(x) \varphi, \psi\right)=\left(\pi_{\lambda, \sigma}(x) \pi_{\lambda, \sigma}(X) \varphi, \psi\right)
$$

and hence

$$
\Omega\left(\pi_{\lambda, \sigma}(x) \varphi, \psi\right)=-\left(|\lambda|^{2}+c_{\sigma}^{2}\right)\left(\pi_{\lambda, \sigma}(x) \varphi, \psi\right) .
$$

Therefore, we can check that

$$
\Omega \operatorname{tr}\left(A \pi_{\lambda, \sigma}(x)^{*}\right)=-\left(|\lambda|^{2}+c_{\sigma}^{2}\right) \operatorname{tr}\left(A \pi_{\lambda, \sigma}(x)^{*}\right) .
$$

This shows that

$$
\frac{\partial}{\partial t} f_{\sigma}(x, t)=\left(\Omega+c_{\sigma}^{2}\right) f_{\sigma}(x, t)
$$

and so we can think of $f_{\sigma}(x, t)$ as generalised heat kernels.

Returning to the symmetric space $G / K$ let us remark that $\Delta$ is nothing but the restriction of $\Omega$ to right $K$-invariant functions. For the rest of this section let us assume $G / K$ is of rank one. In this case $K / M$ is identified with the unit sphere in a Euclidean space. Let $\hat{K}_{M}$ be the set of all class 1 representations of $K$. Then for each $\delta \in \hat{K}_{M}$ there is a unique vector $v_{\delta}$ in the Hilbert space $V_{\delta}$ on which $\delta$ is realised such that $\delta(m) v_{\delta}=v_{\delta}$ for all $m \in M$. Using these representations we can obtain an orthonormal basis $\left\{Y_{\delta j}: 1 \leq j \leq d_{\delta}, \delta \in \hat{K}_{M}\right\}$ for $L^{2}(K / M)$. These functions can be identified as spherical harmonics.

If $f$ is right $K$-invariant then $\hat{f}$ is non zero only on the spherical principal series $\pi_{\lambda, 1}$ and also $\hat{f}(\lambda) Y_{\delta, j}=0$ for all $\delta$ nontrivial. With $Y_{0}(k)=1$ we have

$$
\left(\hat{f}(\lambda) Y_{0}, Y_{\delta j}\right)=\int_{G / K} \int_{K / M} f(x) e^{-(i \lambda+\rho) H(x, b)} Y_{\delta j}(b) d b d x
$$

where we have written $H(x, b)=H\left(x^{-1} b\right)$. The integral

$$
\Phi_{\lambda, \delta}(x)=\int_{K / M} e^{-(i \lambda+\rho) H(x, b)} Y_{\delta, 1}(b) d b
$$

can be explicitly calculated and there exist polynomials $Q_{\delta}(i \lambda+\rho)$ called Kostant polynomials and $K$-biinvariant functions $\varphi_{\lambda, \delta}$ such that

$$
\Phi_{\lambda, \delta}(a)=Q_{\delta}(i \lambda+\rho) \varphi_{\lambda, \delta}(a) .
$$


Moreover, if $x=k a k^{\prime}$,

$$
\int_{K / M} e^{-(i \lambda+\rho) H(x, b)} Y_{\delta, j}(b) d b=\Phi_{\lambda, \delta}(a) Y_{\delta j}(k) .
$$

In view of these formulas we have

$$
\left(\hat{f}(\lambda) Y_{0}, Y_{\delta j}\right)=\int_{A} \int_{K} f(k a) Y_{\delta j}(k) \Phi_{\lambda, \delta}(a) J(a) d a d k .
$$

Writing

$$
f_{\delta, j}(a)=\int_{K} f(k a) Y_{\delta, j}(k) d k
$$

we have

$$
\left(\hat{f}(\lambda) Y_{0}, Y_{\delta j}\right)=Q_{\delta}(i \lambda+\rho) \int_{A} f_{\delta, j}(a) \varphi_{\lambda, \delta}(a) J(a) d a .
$$

It can be shown that $\varphi_{\lambda, \delta}(a)$ are expressible in terms of Jacobi functions which are eigenfunctions of an elliptic differential operator $\Delta_{\delta}$. These operators are related to the Laplace-Beltrami operator. Thus $\left(\hat{f}(\lambda) Y_{0}, Y_{\delta j}\right)$ is $Q_{\delta}(i \lambda+\rho)$ times the Jacobi transform of $f_{\delta, j}(a)$. For all these and more about Jacobi transform we refer to Anker et al. [2], Bray [7] and [39]. Let $p_{t}^{\delta}$ be the heat kernel associated to the operator $\Delta_{\delta}$ which is characterised by

$$
\int_{A} p_{t}^{\delta}(a) \varphi_{\lambda, \delta}(a) J(a) d a=e^{-t\left(\lambda^{2}+\rho^{2}\right)} .
$$

Using inversion formula for Jacobi transform one can obtain very precise upper and lower bounds for $p_{t}^{\delta}$ (see Anker et al. [2]).

We can now state and prove a stronger form of Corollary 3.3.

Corollary 3.5. $\quad$ Let $G / K$ be of rank one and assume that the function $f$ on $G / K$ satisfy the conditions

$$
|f(x)| \leq c(1+|x|)^{N} p_{t}(x),\|\hat{f}(\lambda)\| \leq c(1+|\lambda|)^{N} e^{-t \lambda^{2}} .
$$

Then $f$ is a finite linear combination of functions of the form

$$
f_{\delta}(k a)=Y_{\delta, j}(k) P_{\delta}\left(\Delta_{\delta}\right) p_{t}^{\delta}(a)
$$

where $P_{\delta}$ are polynomials. 
To see this we use the conclusion of Theorem 1.1 with the equation (3.25) to obtain

$$
e^{-t \lambda^{2}} \sum_{m=0}^{N} \lambda^{m}\left(A_{m} Y_{0}, Y_{\delta j}\right)=Q_{\delta}(i \lambda+\rho) \int_{A} f_{\delta, j}(a) \varphi_{\lambda, \delta}(a) J(a) d a .
$$

This equation shows that the polynomial on the left hand side is divisible by $Q_{\delta}(i \lambda+\rho)$ for any $\delta$. There are pair of integers associated to $\delta$ and it is known that $\operatorname{deg} Q_{\delta}(i \lambda+\rho)$ depends on these integers. As these parameters tend to infinity the above equation is possible only if $f_{\delta, j}=0$ for all but finitely many $\delta$. (For the parametrisation of $\delta \in \hat{K}_{M}$ and for the explicit form of $Q_{\delta}(i \lambda+\rho)$ we refer to Johnson-Wallach [18]).

Thus there are polynomials $P_{\delta}$ such that

$$
\int_{A} f_{\delta, j}(a) \varphi_{\lambda, \delta}(a) J(a) d a=P_{\delta}(\lambda) e^{-t \lambda^{2}} .
$$

As the left hand side is an even function of $\lambda$ the same is true of $P_{\delta}$. Since the Jacobi functions are eigenfunctions of $\Delta_{\delta}$ with eigenvalues $\lambda^{2}+\rho^{2}$ we see that on using the inversion formula $f_{\delta j}(a)=P_{\delta}\left(\Delta_{\delta}\right) p_{t}^{\delta}(a)$. This completes the proof of Corollary 3.5.

We conclude this section with two remarks. Even though we have assumed that $G / K$ is of rank one in the above corollary, we believe the same is true in the general case. We have to use Eisenstein integrals and $Q_{\delta}$ matrices in place of $\Phi_{\lambda, \delta}$ and Kostant polynomials. The second remark is about the following result which has been proved in [39].

Theorem 3.6. Let $G / K$ be of rank one and assume that $f$ on $G / K$ satisfies the conditions

$$
|f(x)| \leq c(1+|x|)^{N} p_{t}(x), \quad Q_{\delta}(i \lambda+\rho)^{-1}\left|\left(\hat{f}(\lambda) Y_{0}, Y_{\delta, j}\right)\right| \leq c e^{-t \lambda^{2}}
$$

for every $\delta \in \hat{K}_{M}$ and $1 \leq j \leq d_{\delta}$. Then $f(k a)$ is a finite linear combination of functions of the form $Y_{\delta, j}(k)(\sinh (\log a))^{p}(\cosh (\log a))^{q} p_{t}^{\delta}(a)$.

Note that the hypothesis on $\hat{f}(\lambda)$ is much weaker than the one in Theorem 1.1. So we cannot deduce the above from our Hardy's theorem. In the proof of Theorem 3.6 not only the upper bounds for $p_{t}^{\delta}$ but also the lower bounds play an important role. It is still an open problem if an analogue of Theorem 3.6 is true or not for symmetric spaces of higher rank. 


\section{§4. Hardy's Theorem for $S L(2, \mathbb{R})$}

In this section we specialise to the case of $S L(2, \mathbb{R})$ and show that we can get a complete analogue of Hardy's theorem for the group Fourier transform. For the following results about $S L(2, \mathbb{R})$ and its representation theory we refer to Koornwinder [21]. We closely follow the notations in [22] with minor changes in order to be consistent with the notations used in the previous sections.

Let $G=S L(2, \mathbb{R})$ and consider the Iwasawa decomposition $G=K A N$ with

$$
K=\left\{k_{\theta}=\operatorname{diag}\left(e^{\frac{i}{2} \theta}, e^{-\frac{i}{2} \theta}\right): 0 \leq \theta<4 \pi\right\}
$$

and

$$
A=\left\{a_{r}=\left(\begin{array}{cc}
\cosh r & \sinh r \\
\sinh r & \cosh r
\end{array}\right): r \in \mathbb{R}\right\}
$$

Then $M=\left\{k_{0}, k_{2 \pi}\right\}, \hat{K}$ consists of all characters $\chi_{n}, n \in \frac{1}{2} \mathbb{Z}$ given by $\chi_{n}\left(k_{\theta}\right)=$ $e^{i n \theta}$ and $\hat{M}$ consists of $\chi_{0}$ and $\chi_{\frac{1}{2}}$ restricted to $M$. Let us simply denote $\chi_{0}$ by 0 and $\chi_{\frac{1}{2}}$ by $\frac{1}{2}$ so that $\hat{M}=\left\{0, \frac{1}{2}\right\}$ and the principal series representations of $G$ associated to the above Iwasawa decomposition can be simply written as $\pi_{\lambda, 0}$ and $\pi_{\lambda, \frac{1}{2}}$. These representations are realised on the Hilbert spaces $H(K, 0)$ and $H\left(K, \frac{1}{2}\right)$. For each $\sigma \in \hat{M}$ there exists an orthonormal basis $\left\{e_{j}: j \in \mathbb{Z}+\sigma\right\}$ with the property that

$$
\pi_{\lambda, \sigma}\left(k_{\theta}\right) e_{j}=\chi_{j}\left(k_{\theta}\right) e_{j}=e^{i j \theta} e_{j} .
$$

Let us recall the definition of the Jacobi functions $\varphi_{\lambda}^{(\alpha, \beta)}(r)$. For each $\alpha, \beta, \lambda \in \mathbb{C}$ with $\alpha \neq-1,-2,-3, \ldots$ these functions are defined by

$$
\varphi_{\lambda}^{(\alpha, \beta)}(r)=F\left(\frac{1}{2}(\alpha+\beta+1-i \lambda), \frac{1}{2}(\alpha+\beta+1+i \lambda), \alpha+1,-\sinh ^{2} r\right)
$$

where $F$ is the Gaussian hypergeometric function

$$
F(a, b, c, z)=\sum_{k=0}^{\infty} \frac{(a)_{k}(b)_{k}}{(c)_{k} k !} z^{k}, \quad|z|<1 .
$$

Here $(a)_{0}=1$ and $(a)_{k}=a(a+1) \ldots(a+k-1)$ for $k \geq 1$. The importance of the Jacobi function arises from the fact that the matrix coefficients of $\pi_{\lambda, \sigma}$ are expressible in terms of $\varphi_{\lambda}^{(\alpha, \beta)}(r)$. In fact, for $\sigma \in \hat{M}$ and $m, j \in \mathbb{Z}+\sigma$

$$
\left(\pi_{\lambda, \sigma}\left(a_{r}\right) e_{j}, e_{m}\right)=\frac{c_{\lambda, \sigma}(m, j)}{(|m-j|) !}(\sinh r)^{|m-j|}(\cosh r)^{m+j} \varphi_{\lambda}^{(|m-j|, m+j)}(r)
$$


where the functions $c_{\lambda, \sigma}(m, j)$ are given by

$$
c_{\lambda, \sigma}(m, j)=\left(-\frac{i}{2} \lambda+j+\frac{1}{2}\right)_{m-j}
$$

if $m \geq j$ and

$$
c_{\lambda, \sigma}(m, j)=\left(-\frac{i}{2} \lambda-j+\frac{1}{2}\right)_{j-m}
$$

if $m \leq j$. Note that $c_{\lambda, \sigma}(m, m)=1$ for all $m \in \mathbb{Z}+\sigma$.

With the above preparations we can proceed to a proof of Theorem 1.3. From Theorem 1.1 we already know that

$$
\hat{f}(\lambda, \sigma)=e^{-t \lambda^{2}} A_{\sigma}, \quad \lambda \in \mathbb{C}
$$

where $A_{\sigma}$ is a bounded linear operator on $H(K, \sigma)$. If $\left\{e_{m}: m \in \mathbb{Z}+\sigma\right\}$ is the basis described above we have

$$
e^{-t \lambda^{2}}\left(A_{\sigma} e_{j}, e_{m}\right)=\int_{G} f(x)\left(\pi_{\lambda, \sigma}(x) e_{j}, e_{m}\right) d x .
$$

Since the Haar measure on $G$ is a constant multiple of $(\sinh 2 r) d r d \theta d \varphi$ using (4.1) we get

$e^{-t \lambda^{2}}\left(A_{\sigma} e_{j}, e_{m}\right)=c \int_{0}^{\infty} \int_{0}^{4 \pi} \int_{0}^{4 \pi} f\left(k_{\theta} a_{r} k_{\varphi}\right) e^{i m \theta} e^{i j \varphi}\left(\pi_{\lambda, \sigma}\left(a_{r}\right) e_{j}, e_{m}\right)(\sinh 2 r) d r d \theta d \varphi$.

In view of (4.2), (4.3) and (4.4) the right hand side vanishes for certain values of $\lambda$ when $m \neq j$ which forces $\left(A_{\sigma} e_{j}, e_{m}\right)=0$ whenever $m \neq j$. And when $m=j$

$$
e^{-t \lambda^{2}}\left(A_{\sigma} e_{m}, e_{m}\right)=c \int_{0}^{\infty} f_{m}(r) \varphi_{\lambda}^{(0,2 m)}(r)(2 \sinh r)(2 \cosh r)^{2 m+1} d r
$$

where we have defined

$$
f_{m}(r)=\int_{0}^{4 \pi} \int_{0}^{4 \pi} f\left(k_{\theta} a_{r} k_{\varphi}\right) e^{i m(\theta+\varphi)} d \theta d \varphi .
$$

Recall that the Jacobi transform $J_{\alpha, \beta}$ of type $(\alpha, \beta)$ is defined by

$$
J_{\alpha, \beta} g(\lambda)=\int_{0}^{\infty} g(r) \varphi_{\lambda}^{(\alpha, \beta)}(r)(2 \sinh r)^{2 \alpha+1}(2 \cosh r)^{2 \beta+1} d r .
$$


We have inversion and Plancherel theorems for the Jacobi transform. The function $h_{t}^{(\alpha, \beta)}$ defined by the equation

$$
e^{-t\left(\lambda^{2}+\rho^{2}\right)}=\int_{0}^{\infty} h_{t}^{(\alpha, \beta)}(r) \varphi_{\lambda}^{(\alpha, \beta)}(r)(2 \sinh r)^{2 \alpha+1}(2 \cosh r)^{2 \beta+1} d r
$$

where $\rho=\alpha+\beta+1$ is called the heat kernel of type $(\alpha, \beta)$. It is the heat kernel associated to the Jacobi differential operator with parameters $\alpha$ and $\beta$. In particular, $h_{t}^{(0,0)}(r)$ is a constant multiple of the heat kernel $p_{t}\left(a_{r}\right)$ for the Laplace-Beltrami operator on $S L(2, \mathbb{R})$. We make use of the following estimates proved in Anker et al. [2].

Theorem 4.1. Let $\alpha \geq \beta$ be integers with $2 \beta+1 \geq 0$. Then there are constants $c_{1}$ and $c_{2}$ such that

$$
c_{1} t^{-\frac{3}{2}} e^{-\rho^{2} t} H_{t}^{(\alpha, \beta)}(r) \leq h_{t}^{(\alpha, \beta)}(r) \leq c_{2} t^{-\frac{3}{2}} e^{-\rho^{2} t} H_{t}^{(\alpha, \beta)}(r)
$$

where $\rho=\alpha+\beta+1$ and

$$
H_{t}^{(\alpha, \beta)}(r)=(1+r)\left(1+\frac{1+r}{t}\right)^{\alpha-\frac{1}{2}} e^{-\rho r-\frac{r^{2}}{4 t}} .
$$

The equation (4.6) shows that $f_{m}(r)(\cosh r)^{-2 m}$ is a constant multiple of $h_{t}^{(0,2 m)}(r)$. We cannot directly appeal to Theorem 4.1 to estimate $h_{t}^{(0,2 m)}(r)$ as the conditions on $\alpha$ and $\beta$ are not satisfied. However, we can express $h_{t}^{(0,2 m)}$ in terms of $h_{t}^{(n, 0)}$ with $n \in \mathbb{N}$ and then use Theorem 4.1 to estimate $h_{t}^{(0,2 m)}$. In order to do this we make use of the following formula which relates Jacobi transforms of different types. Let us define

$$
w_{\alpha, \beta}(r)=(2 \sinh r)^{2 \alpha+1}(2 \cosh r)^{2 \beta+1}
$$

so that

$$
J_{\alpha, \beta} f(\lambda)=\int_{0}^{\infty} f(r) \varphi_{\lambda}^{(\alpha, \beta)}(r) w_{\alpha, \beta}(r) d r .
$$

Then for any $n \in \mathbb{N}$ (see Koornwinder [22])

$$
\begin{aligned}
\text { (4.10) } & J_{\alpha, \beta} f(\lambda) \\
= & \frac{(-1)^{n}}{2^{4 n}(\alpha+1)_{n}} \int_{0}^{\infty}\left(\frac{1}{\sinh 2 r} \frac{d}{d r}\right)^{n} f(r) \varphi_{\lambda}^{(\alpha+n, \beta+n)}(r) w_{\alpha+n, \beta+n}(r) d r .
\end{aligned}
$$

Here $\alpha$ is assumed to be different from $-1,-2,-3, \ldots$. 
Lemma 4.2. For all $n \in \mathbb{I}$ and $\sigma \in\left\{0, \frac{1}{2}\right\}$

$$
\left(\frac{1}{\sinh 2 r} \frac{d}{d r}\right)^{2 n \pm 2 \sigma}\left(f_{ \pm n+\sigma}(r)(\cosh r)^{2 n \pm 2 \sigma}\right)=c_{n, \sigma} h_{t}^{(2 n \pm 2 \sigma, 0)}(r)
$$

for some constants $c_{n, \sigma}$.

Proof. First assume that $m=-n+\sigma, n \in \mathbb{N}$ and consider the equation

$$
\begin{aligned}
& \int_{0}^{\infty} f_{-n+\sigma}(r)(\cosh r)^{-2 n+2 \sigma} \varphi_{\lambda}^{(0,-2 n+2 \sigma)}(r) w_{0,0}(r) d r \\
= & c_{-n+\sigma} e^{-t \lambda^{2}} .
\end{aligned}
$$

Using the formula (4.10) we can rewrite this as

$$
\begin{aligned}
& \int_{0}^{\infty} f_{-n+\sigma}(r)(\cosh r)^{2 n-2 \sigma} \varphi_{\lambda}^{(0,-2 n+2 \sigma)}(r) w_{0,-2 n+2 \sigma}(r) d r \\
& =d_{n} \int_{0}^{\infty}\left(\frac{1}{\sinh 2 r} \frac{d}{d r}\right)^{2 n-2 \sigma}\left(f_{-n+\sigma}(r)(\cosh r)^{2 n-2 \sigma}\right) \\
& \quad \times \varphi_{\lambda}^{(2 n-2 \sigma, 0)}(r) w_{2 n-2 \sigma, 0}(r) d r
\end{aligned}
$$

which is a constant multiple of $e^{-t \lambda^{2}}$. This shows that

$$
\left(\frac{1}{\sinh 2 r} \frac{d}{d r}\right)^{2 n-2 \sigma}\left(f_{-n+\sigma}(r)(\cosh r)^{2 n-2 \sigma}\right)=c_{n, \sigma} h_{t}^{(2 n-2 \sigma, 0)}(r)
$$

for all $n \in \mathbb{N}$. $\left(n \geq 1\right.$ if $\sigma=\frac{1}{2}$.)

Next assume that $m=n+\sigma, n \in \mathbb{N}$. In this case we use the formula

$$
\varphi_{\lambda}^{(\alpha, \beta)}(r)=(2 \cosh r)^{-2 \beta} \varphi_{\lambda}^{(\alpha,-\beta)}(r) .
$$

This formula shows that

$$
\begin{aligned}
& \int_{0}^{\infty} f_{n+\sigma}(r)(\cosh r)^{2 n+2 \sigma} \varphi_{\lambda}^{(0,2 n+2 \sigma)}(r) w_{0,0}(r) d r \\
= & d_{n} \int_{0}^{\infty} f_{n+\sigma}(r)(\cosh r)^{-2 n-2 \sigma} \varphi_{\lambda}^{(0,-2 n-2 \sigma)}(r) w_{0,0}(r) d r \\
= & d_{n} \int_{0}^{\infty} f_{n+\sigma}(r)(\cosh r)^{2 n+2 \sigma} \varphi_{\lambda}^{(0,-2 n-2 \sigma)}(r) w_{0,-2 n-2 \sigma}(r) d r \\
= & d_{n} \int_{0}^{\infty}\left(\frac{1}{\sinh 2 r d r}\right)^{2 n+2 \sigma}\left(f_{n+\sigma}(r)(\cosh r)^{2 n+2 \sigma}\right) \varphi_{\lambda}^{(2 n+2 \sigma, 0)}(r) w_{2 n+2 \sigma, 0}(r) d r .
\end{aligned}
$$

This proves that

$$
\left(\frac{1}{\sinh 2 r} \frac{d}{d r}\right)^{2 n+2 \sigma}\left(f_{n+\sigma}(r)(\cosh r)^{2 n+2 \sigma}\right)=c_{n, \sigma} h_{t}^{(2 n+2 \sigma, 0)}(r) .
$$


Therefore, we only need to estimate the functions $f_{n}$ defined by the equation

$$
\left(\frac{1}{\sinh 2 r} \frac{d}{d r}\right)^{2 n}\left(f_{n}(r)(\cosh r)^{2 n}\right)=c_{n} h_{t}^{(2 n, 0)}(r) .
$$

Let $W_{\mu}^{2}$ be the Weyl fractional integral operator defined by

$$
W_{\mu}^{2} g(r)=\frac{1}{\Gamma(\mu)} \int_{r}^{\infty}(\cosh 2 s-\cosh 2 r)^{\mu-1} g(s)(\sinh 2 s) d s .
$$

Then we know that (see Koornwinder [22])

$$
W_{-2 n}^{2}=\left(\frac{1}{\sinh 2 r} \frac{d}{d r}\right)^{2 n}
$$

and therefore,

(4.14) $f_{n}(r)(\cosh r)^{2 n}=c_{n} \int_{r}^{\infty}(\sinh 2 s)(\cosh 2 s-\cosh 2 r)^{2 n-1} h_{t}^{(2 n, 0)}(s) d s$.

We can now establish the following estimates.

Proposition 4.3. For every $n \in \mathbb{N}$ there are constants $c_{1}$ and $c_{2}$ depending on $n$ such that

$$
c_{1}\left(\frac{r}{r+1}\right)^{2 n-1} h_{t}^{(0,0)}(r) \leq\left|f_{n}(r)\right| \leq c_{2} h_{t}^{(0,0)}(r) .
$$

Proof. We write $f \sim g$ if $\frac{f(r)}{g(r)}$ is bounded above and below for all $r>0$. From Theorem 4.1 we know that

$$
h_{t}^{(2 n, 0)}(r) \sim(1+r)^{2 n+\frac{1}{2}} e^{-(2 n+1) r} e^{-\frac{r^{2}}{4 t}} .
$$

As we are fixing $t$ we ignore the dependence of constants on $t$. The integral on the right hand side of (4.14) behaves like the integral

$$
\int_{r}^{\infty}(\sinh 2 s)(\cosh 2 s-\cosh 2 r)^{2 n-1}(1+s)^{2 n+\frac{1}{2}} e^{-(2 n+1) s} e^{-\frac{s^{2}}{4 t}} d s .
$$

By the change of variables $s \rightarrow \frac{s}{2}+r$ the above integral is transformed into a constant multiple of

$$
e^{-(2 n+1) r} e^{-\frac{r^{2}}{4 t}} I(r)
$$


where $I(r)$ is defined to be the integral

$$
\begin{gathered}
\int_{0}^{\infty}(\sinh (s+2 r))(\cosh (s+2 r)-\cosh 2 r)^{2 n-1} \\
\quad \times(2+s+2 r)^{2 n+\frac{1}{2}} e^{-\frac{1}{2}(2 n+1) s} e^{-\frac{s^{2}}{16 t}} e^{-\frac{r s}{4 t}} d s .
\end{gathered}
$$

As in Anker et al. [2] we make use of the estimates $\sinh v \sim \frac{v}{1+v} e^{v},(\cosh v-$ $\cosh (v-w)) \sim(\sinh v) \frac{w}{1+w}$ valid for $v \geq w \geq 0$. In view of this $I(r) \sim e^{4 n r} I_{1}(r)$ where $I_{1}(r)$ is the integral

$$
\int_{0}^{\infty}\left(\frac{s+2 r}{1+s+2 r}\right)^{2 n}\left(\frac{s}{s+1}\right)^{2 n-1}(2+s+2 r)^{2 n+\frac{1}{2}} e^{\frac{1}{2}(2 n-1) s} e^{-\frac{s^{2}}{16 t}} e^{-\frac{r s}{4 t}} d s .
$$

As $(2+s+2 r) \leq 2(1+s)(1+r)$ and $(1+s)^{\frac{3}{2}} e^{\frac{1}{2}(2 n-1) s-\frac{s^{2}}{16 t}+\frac{s}{4 t}}$ is bounded

$$
I_{1}(r) \leq C(1+r)^{2 n+\frac{1}{2}} \int_{0}^{\infty} s^{2 n-1} e^{-\frac{(r+1)}{4 t} s} d s \leq C(1+r)^{\frac{1}{2}} .
$$

Therefore,

$$
(\cosh r)^{2 n}\left|f_{n}(r)\right| \leq C_{n} e^{(2 n-1) r} e^{-\frac{r^{2}}{4 t}}(1+r)^{\frac{1}{2}}
$$

and this establishes the upper bound

$$
\left|f_{n}(r)\right| \leq C e^{-r-\frac{r^{2}}{4 t}}(1+r)^{\frac{1}{2}}
$$

and hence we have the estimate $\left|f_{n}(r)\right| \leq C h_{t}^{(0,0)}(r)$.

In order to get a lower bound, observe that $\frac{s+2 r}{1+s+2 r}$ is an increasing function of $s \geq 0$ so that $\frac{s+2 r}{1+s+2 r} \geq\left(\frac{2 r}{1+2 r}\right)$. If $r \geq 1$ and $0<s \leq \frac{1}{r}$ we have $e^{-a r s} \geq e^{-a}$ and $e^{-b s^{2}} \geq e^{-b}$ for any $a, b>0$. Therefore,

$$
\begin{aligned}
I_{1}(r) & \geq c(1+r)^{2 n+\frac{1}{2}}\left(\frac{r}{1+r}\right)^{2 n} \int_{0}^{\frac{1}{r}}\left(\frac{s}{1+s}\right)^{2 n-1} d s \\
& \geq c(1+r)^{\frac{1}{2}} r^{2 n} \cdot\left(1+\frac{1}{r}\right)^{-2 n+1} \int_{0}^{\frac{1}{r}} s^{2 n-1} d s
\end{aligned}
$$

which gives the estimate $c(1+r)^{\frac{1}{2}}\left(\frac{r}{1+r}\right)^{2 n-1}$. This completes the proof of Proposition 4.3.

Returning to the proof of Theorem 1.3 we have shown that $\left(A_{\sigma} e_{j}, e_{m}\right)=0$ for $j \neq m$ and so $\hat{f}(\lambda, \sigma)$ is diagonalised by the orthonormal basis $\left\{e_{m}: m \in\right.$ $\mathbb{Z}+\sigma\}$. Define a function $g$ by

$$
g\left(k_{\theta} a_{r} k_{\varphi}\right)=\sum_{m=-\infty}^{\infty}\left(f_{m}(r) e^{-i m(\theta+\varphi)}+f_{m+\frac{1}{2}}(r) e^{-i\left(m+\frac{1}{2}\right)(\theta+\varphi)}\right) .
$$


Let $h_{m}\left(k_{\theta} a_{r} k_{\varphi}\right)=f_{m}(r) e^{-i m(\theta+\varphi)}$ and define $f_{m+\frac{1}{2}}(r)$ similarly so that

$$
g(x)=\sum_{-\infty}^{\infty}\left(h_{m}(x)+h_{m+\frac{1}{2}}(x)\right) .
$$

It is easily seen that $\hat{h}_{m}\left(\lambda, \frac{1}{2}\right)=\hat{h}_{m+\frac{1}{2}}(\lambda, 0)=0$ and $\left(\hat{h}_{m}(\lambda, 0) e_{m}, e_{j}\right)=0$ for $m, j \in \mathbb{Z}, m \neq j,\left(\hat{h}_{m+\frac{1}{2}}\left(\lambda, \frac{1}{2}\right) e_{m}, e_{j}\right)=0$ for $m, j \in \mathbb{Z}+\frac{1}{2}, m \neq j$. Further,

$$
\left(\widehat{h}_{m}(\lambda, 0) e_{m}, e_{m}\right)=c_{m} e^{-t \lambda^{2}}, \quad m \in \mathbb{Z}
$$

and also

$$
\left(\widehat{h}_{m+\frac{1}{2}}\left(\lambda, \frac{1}{2}\right) e_{m}, e_{m}\right)=c_{m} e^{-t \lambda^{2}}, \quad m \in \mathbb{Z}+\frac{1}{2}
$$

Thus we see that all the functions $h_{m}, h_{m+\frac{1}{2}}$ satisfy both conditions of the Theorem 1.3. Moreover, $\hat{f}(\lambda, \sigma)=\hat{g}(\lambda, \sigma)$ for all $\lambda \in \mathbb{C}$ and $\sigma \in \hat{M}$. This shows that the Fourier transform of $f-g$ is supported on the discrete series representations of $S L(2, \mathbb{R})$. We can now argue as in Sitaram-Sundari [35] using the estimate on $p_{t}(x)$ to show that $f-g$ is actually zero a.e. This proves that

$$
f\left(k_{\theta} a_{r} k_{\varphi}\right)=\sum_{-\infty}^{\infty}\left(f_{m}(r) e^{-i m(\theta+\varphi)}+f_{m+\frac{1}{2}}(r) e^{-i\left(m+\frac{1}{2}\right)(\theta+\varphi)}\right) .
$$

This completes the proof of Theorem 1.3.

\section{$\S 5 . \quad$ Gelfand-Shilov and Beurling Theorems}

In this section we prove Theorems 1.4 and 1.5. We have seen that the proof of Hardy's theorem depended heavily on the complex analytic Lemma 3.1. In a similar way, the proof of Beurling's theorem given in Bonami et al. [6] depends on the following lemma on entire functions of order 2. The following lemma, in one dimension, is implicit in the work of Hörmander [17]. For the next two lemmas we refer to $[6]$.

Lemma 5.1. Let $g(w)$ be an entire function on $\mathbb{C}^{n}$ of order 2. Assume that for every $\alpha \in\left(0, \frac{\pi}{2}\right]$ the entire function

$$
G_{\alpha}(z, \xi)=\int_{0}^{z \xi_{1}} \cdots \int_{0}^{z \xi_{n}} g(w) \overline{g\left(e^{i \alpha} \bar{w}\right)} d w
$$

satisfies the estimate

$$
\left|G_{\alpha}(z, \xi)\right| \leq c(1+|z||\xi|)^{N}
$$


where $c$ is independent of $\alpha$. Then $g(z) g(i z)=R(z)$ where $R(z)$ is a polynomial of degree $\leq N-n$.

This lemma is proved by a repeated application of Phragmen-Lindelöf maximum principle. From the fact that $g(w) g(i w)$ is a polynomial the following lemma allows us to completely characterise the function $g$.

Lemma 5.2. Let $g$ be an entire function of order 2 on $\mathbb{C}^{n}$ such that $g(z) g(i z)$ is a polynomial of degree $N$. Then there exists a polynomial $P(z)$ of degree $\leq \frac{N}{2}$ and a homogeneous polynomial $Q(z)$ of degree 2 such that $g(z)=$ $P(z) e^{Q(z)}$.

With these two lemmas at hand we now embark on a proof of Theorem 1.4. For $\varphi, \psi \in H(K, \sigma)$ consider the function

$$
F(\lambda)=(\hat{f}(\lambda, \sigma) \varphi, \psi), \lambda \in \mathfrak{a}^{*} .
$$

We first show that $F(\lambda)$ extends to an entire function of order 2 which satisfies the conditions of Lemma 5.1. Then we appeal to Lemma 5.2 to prove the following.

Proposition 5.3. $\quad$ Under the hypothesis of Theorem 1.4 the function $F(\lambda)$ defined in (5.1) can be written as $F(\lambda)=P(\lambda) e^{-(E \lambda, \lambda)}$ where $P(\lambda)=$ $P(\lambda, \varphi, \psi)$ is a polynomial and $E=E(\varphi, \psi)$ is a complex symmetric matrix.

Proof. We first show that $F(\lambda)$ extends to an entire function of $\lambda \in \mathfrak{a}_{\mathbb{C}}^{*} \cong$ $\mathbb{C}^{\ell}$. Using the estimate

$$
\left|\left(\pi_{\lambda, \sigma}(x) \varphi, \psi\right)\right| \leq c\|\varphi\|_{2}\|\psi\|_{2} e^{|\operatorname{Im}(\lambda)||\log a|}
$$

we have

$$
|F(\lambda)| \leq c\|\varphi\|_{2}\|\psi\|_{2} \int_{G}\left|f\left(k a k^{\prime}\right)\right| e^{|\operatorname{Im}(\lambda)||\log a|} e^{2 \rho(\log a)} d a d k d k^{\prime} .
$$

Since $a b \geq 1$,

$$
\begin{aligned}
|\operatorname{Im}(\lambda)||\log a| & \leq \frac{1}{2} a \cdot 2 b \cdot|\operatorname{Im}(\lambda)||\log a| \\
& \leq \frac{1}{p}\left(\frac{1}{2} a|\log a|\right)^{p}+\frac{1}{q}(2 b|\operatorname{Im}(\lambda)|)^{q} .
\end{aligned}
$$

Using this we obtain

$$
|F(\lambda)| \leq c\|\varphi\|_{2}\|\psi\|_{2} e^{\frac{1}{q}(2 b|\operatorname{Im}(\lambda)|)^{q}} I
$$


where $I$ is the integral

$$
\begin{aligned}
I & =\int_{G}\left|f\left(k a k^{\prime}\right)\right| e^{\frac{1}{p}\left(\frac{1}{2} a|\log a|\right)^{p}} e^{2 \rho(\log a)} d a d k d k^{\prime} \\
& \leq \int_{G} \frac{\left|f\left(k a k^{\prime}\right)\right|}{(1+|\log a|)^{N}} e^{\frac{a^{p}}{p}|\log a|^{p}} e^{2 \rho(\log a)} d a d k d k^{\prime}<\infty .
\end{aligned}
$$

Hence, we see that $F(\lambda)$ extends to an entire function satisfying

$$
|F(\lambda)| \leq c\|\varphi\|_{2}\|\psi\|_{2} e^{\beta|\operatorname{Im}(\lambda)|^{2}} .
$$

Next we want to show that

$$
\int_{|\lambda| \leq R}|F(\lambda)||F(i \lambda)| d \lambda \leq c(1+R)^{2 N} .
$$

To prove this estimate, we consider

$$
\begin{aligned}
\int_{|\lambda| \leq R}|F(\lambda)||F(i \lambda)| d \lambda & \leq \int_{|\lambda| \leq R} \int_{G}|F(\lambda)||f(x)|\left|\left(\pi_{i \lambda, \sigma}(x) \varphi, \psi\right)\right| d x d \lambda \\
& \leq c\|\varphi\|_{2}\|\psi\|_{2} \int_{|\lambda| \leq R} \int_{G}|F(\lambda)||f(x)| e^{|\lambda||x|} d x d \lambda .
\end{aligned}
$$

Taking $d=\left(2 R p a^{-p}\right)^{\frac{1}{p-1}}$ we split the integral over $G$ into two parts and estimate them separately. As $p \geq 2, d \leq c R$ and so

$$
\begin{aligned}
& \int_{|\lambda| \leq R} \int_{|x| \leq d}|F(\lambda)||f(x)| e^{|\lambda||x|} d x d \lambda \\
\leq & c(1+R)^{2 N} \int_{G} \int_{\mathfrak{a}^{*}} \frac{|F(\lambda)|}{(1+|\lambda|)^{N}} \frac{|f(x)|}{(1+|x|)^{N}} e^{\frac{a^{p}}{p}|x|^{p}} e^{\frac{b^{q}}{q}|\lambda|^{q}} d \lambda d x \\
\leq & c\|\varphi\|_{2}\|\psi\|_{2}(1+R)^{2 N}
\end{aligned}
$$

where we have used $|F(\lambda)| \leq\|\hat{f}(\lambda, \sigma)\|\|\varphi\|_{2}\|\psi\|_{2}$.

For the second integral taken over $|x|>d$ we note that $|x|^{p-1}>2 R p a^{-p}$ and so for $|\lambda| \leq R,|\lambda||x| \leq R|x| \leq \frac{1}{2 p} a^{p}|x|^{p}$. Therefore,

$$
\begin{aligned}
& \int_{|\lambda| \leq R} \int_{|x|>d}|F(\lambda)||f(x)| e^{|\lambda||x|} d x d \lambda \\
\leq & c(1+R)^{N} \int_{\mathfrak{a}^{*}} \int_{G} \frac{|F(\lambda)|}{(1+|\lambda|)^{N}}|f(x)| e^{\frac{1}{2 p} a^{p}|x|^{p}} d x d \lambda \\
\leq & c(1+R)^{N}\|\varphi\|_{2}\|\psi\|_{2} .
\end{aligned}
$$

Thus we have proved the estimate

$$
\int_{|\lambda| \leq R}|F(\lambda)||F(i \lambda)| d \lambda \leq C_{\varphi, \psi}(1+R)^{2 N} .
$$


In order to apply Lemma 5.1 we need to estimate the integral

$$
\int_{0}^{z \xi_{1}} \ldots \int_{0}^{z \xi_{\ell}} F(w) \overline{F\left(e^{i \alpha} \bar{w}\right)} d w=z^{\ell} \int_{0}^{\xi_{1}} \ldots \int_{0}^{\xi_{\ell}} F(z w) \overline{F\left(e^{i \alpha} \bar{z} w\right)} d w
$$

for every $\alpha \in\left(0, \frac{\pi}{2}\right]$ :

Assuming $\alpha<\frac{\pi}{2}$, the integral

$$
z^{\ell} \int_{0}^{\xi_{1}} \ldots \int_{0}^{\xi_{\ell}} F(z w) \overline{F\left(e^{i \alpha} \bar{z} w\right)} d w
$$

can be estimated for $z \in \mathbb{R}$ and $z \in e^{i \alpha} \mathbb{R}$ as above leading to

$$
\left|\int_{0}^{z \xi_{1}} \cdots \int_{0}^{z \xi_{\ell}} F(w) \overline{F\left(e^{i \alpha} \bar{w}\right)} d w\right| \leq c(1+|z||\xi|)^{2 N} .
$$

By Phragmen-Lindelöf the same estimate is valid for $z \in e^{i \alpha / 2} \mathbb{R}$. Hence

$$
\int_{0}^{x \xi_{1}} \cdots \int_{0}^{x \xi_{\ell}}\left|F\left(e^{i \alpha / 2} w\right)\right|^{2} d w \leq c(1+|x||\xi|)^{2 N} .
$$

As the estimate is uniform in $\alpha$ we get the same for $\alpha=\frac{\pi}{2}$. This means that the function

$$
G_{\xi}(z)=G(z \xi)=\int_{0}^{z \xi_{1}} \cdots \int_{0}^{z \xi_{\ell}} F(w) F(i w) d w
$$

satisfies the estimate

$$
\left|G_{\xi}(z)\right| \leq c(1+|z||\xi|)^{2 N}
$$

for $z \in \mathbb{R}, i \mathbb{R}$ and $e^{i \frac{\pi}{4}} \mathbb{R}$. One more application of Phragmen-Lindelöf shows that $G_{\xi}(z)$ is an entire function of polynomial growth. Then proceeding as in [6] we get

$$
F(w) F(i w)=R(w)
$$

where $R$ is a polynomial of degree $\leq 2 N-\ell$.

We can now appeal to Lemma 5.2 to arrive at

$$
F(\lambda)=P(\lambda) e^{Q(\lambda)}, \operatorname{deg} P \leq N-\frac{\ell}{2}
$$

Writing $Q(\lambda)=-(E \lambda, \lambda)$, with $E$ symmetric we complete the proof of Proposition 5.3. 
The polynomial $P(\lambda)$ and the matrix $E$ both depend on $\sigma$ and the functions $\varphi, \psi \in H(K, \sigma)$. Thus $P(\lambda)=P_{\sigma}(\lambda, \varphi, \psi)$ and $E=E_{\sigma}(\varphi, \psi)$. First we want to show that $E_{\sigma}$ is independent of $\varphi$ and $\psi$. To prove this define

$$
p(s, t, \lambda)=P_{\sigma}(\lambda, s \varphi, t \psi), d(s, t)=E_{\sigma}(s \varphi, t \psi) .
$$

Then recalling the definition of $F(\lambda)$ we have the equation

$$
s t(\hat{f}(\lambda, \sigma) \varphi, \psi)=p(s, t, \lambda) e^{-(d(s, t) \lambda, \lambda)} .
$$

We observe that $p(0, t, \lambda)=0$ and hence

$$
t(\hat{f}(\lambda, \sigma) \varphi, \psi)=s^{-1}(p(s, t, \lambda)-p(0, t, \lambda)) e^{-(d(s, t) \lambda, \lambda)} .
$$

This shows that $p(s, t, \lambda)$ is differentiable at $s=0$ and

$$
t(\hat{f}(\lambda, \sigma) \varphi, \psi)=\partial_{s} p(0, t, \lambda) e^{-(d(0, t) \lambda, \lambda)} .
$$

Repeating the argument with $t$ we obtain

$$
(\hat{f}(\lambda, \sigma) \varphi, \psi)=\partial_{t} \partial_{s} p(0,0, \lambda) e^{-(d(0,0) \lambda, \lambda)} .
$$

Since $d(0,0)=E_{\sigma}(0,0)$ we obtain the equation

$$
(\hat{f}(\lambda, \sigma) \varphi, \psi)=P_{\sigma}(\lambda, \varphi, \psi) e^{-\left(E_{\sigma} \lambda, \lambda\right)}
$$

where $E_{\sigma}$ is independent of $\varphi$ and $\psi$.

From the above expression it is clear that $P_{\sigma}(\lambda, \varphi, \psi)$ is linear in $\varphi$ and $\psi$. Writing

$$
P_{\sigma}(\lambda, \varphi, \psi)=\sum_{|\alpha| \leq N} C_{\sigma, \alpha}(\varphi, \psi) \lambda^{\alpha}
$$

it is clear that $C_{\sigma, \alpha}(\varphi, \psi)$ are sesquilinear forms on $H(K, \sigma)$. Actually they are bounded. This can be proved if we use the estimate (5.2) along with Cauchy's integral formula. Hence

$$
\left|C_{\sigma, \alpha}(\varphi, \psi)\right| \leq C_{\sigma, \alpha}\|\varphi\|_{2}\|\psi\|_{2}
$$

where the constants $C_{\sigma, \alpha}$ are independent of $\varphi$ and $\psi$. Therefore, there are bounded linear operators $A_{\sigma, \alpha}$ such that $C_{\sigma, \alpha}(\varphi, \psi)=\left(A_{\sigma, \alpha} \varphi, \psi\right)$. This leads to the equation

$$
\hat{f}(\lambda, \sigma)=e^{-\left(E_{\sigma} \lambda, \lambda\right)}\left(\sum_{|\alpha| \leq N} \lambda^{\alpha} A_{\sigma, \alpha}\right)
$$

as operators on $H(K, \sigma)$. 
In order to complete the proof of Theorem 1.4 we need to consider several cases. First consider the case $p=q=2$. Let us write $E_{\sigma}=A_{\sigma}+i B_{\sigma}$ where $A_{\sigma}$ and $B_{\sigma}$ are real symmetric. The hypothesis on $\hat{f}(\lambda, \sigma)$ shows that $A_{\sigma}$ has to be positive definite. We claim that $\left(A_{\sigma} \lambda, \lambda\right) \geq \frac{1}{2} b^{2}|\lambda|^{2}$ for $\lambda \in \mathfrak{a}^{*}$. To see this let $S_{\sigma}$ be an orthogonal matrix such that $S_{\sigma} A_{\sigma} S_{\sigma}^{-1}=\operatorname{diag}\left(b_{1}, b_{2}, \ldots, b_{\ell}\right)$. Then we have

$$
\int_{\mathbb{R}^{\ell}} \frac{\left|P_{\sigma}\left(S_{\sigma}^{-1} \lambda, \varphi, \psi\right)\right|}{(1+|\lambda|)^{N}} e^{-\sum_{j=1}^{\ell} b_{j} \lambda_{j}^{2}} e^{\frac{1}{2} b^{2}|\lambda|^{2}} d \lambda<\infty .
$$

This certainly implies that $b_{j} \geq \frac{1}{2} b^{2}$ for every $j$. Therefore, $\left(S_{\sigma} A_{\sigma} S_{\sigma}^{-1} \lambda, \lambda\right) \geq$ $\frac{1}{2} b^{2}|\lambda|^{2}$ which implies $A_{\sigma} \geq \frac{1}{2} b^{2} I$ as desired.

When $p=q=2$ and $a b>1$ we want to show that $(\hat{f}(\lambda, \sigma) \varphi, \psi)=0$ for all $\varphi, \psi \in H(K, \sigma)$. Since $a b>1$ we can find $a>\alpha, b>\beta$ with $\alpha \beta>1$. Then consider

$$
\begin{aligned}
& \int_{|\lambda| \leq R}|F(\lambda)||F(i \lambda)| d \lambda \\
\leq & c \int_{|\lambda| \leq R} \int_{G}|F(\lambda)||f(x)| e^{|\lambda||x|} d x d \lambda \\
\leq & c \int_{|\lambda| \leq R} \int_{G}|F(\lambda)||f(x)| e^{\frac{\alpha^{2}}{2}|x|^{2}} e^{\frac{\beta^{2}}{2}|\lambda|^{2}} d x d \lambda \leq c
\end{aligned}
$$

uniformly in $R$. Therefore, we can conclude that the function $G_{\alpha}(z \xi)$ is a constant which implies that $F(\lambda) F(i \lambda)=0$. Therefore, when $p=q=2$ and $a b>1$ we get $\hat{f}(\lambda, \sigma)=0$ for all $\sigma \in \hat{M}$ and $\lambda \in \mathfrak{a}^{*}$.

Finally, we have to deal with the case $1<q<2$. In this case, we can choose $\beta>b$ and $\alpha<a$ such that $\alpha \beta \geq 1$. As before,

$$
\int_{|\lambda| \leq R}|F(\lambda)||F(i \lambda)| d \lambda \leq c \int_{|\lambda| \leq R} \int_{G}|F(\lambda)||f(x)| e^{\frac{\alpha^{p}}{p}|x|^{p}} e^{\frac{\beta^{q}}{q}|\lambda|^{q}} d x d \lambda \leq c
$$

uniformly in $R$ since $\alpha<a$ and $F(\lambda)=P(\lambda) e^{-\left(E_{\sigma} \lambda, \lambda\right)}$ with $R e E_{\sigma}$ positive definite. Hence $\hat{f}(\lambda, \sigma)=0$ in this case also. This completes the proof of Theorem 1.4.

We remark that when $p=q=2, a b=1$ some of the eigenvalues $b_{j}$ of $E_{\sigma}$ should be equal to $\frac{1}{2} b^{2}$. For, if $b_{j}>\frac{1}{2} b^{2}$ for all $j$ then proceeding as above we can conclude that $\hat{f}(\lambda, \sigma)=0$. In the rank one case this gives us the following result. 
Corollary 5.4. Assume that $G$ is of rank 1. Then the conclusion of Theorem 1.4 reads as

$$
\hat{f}(\lambda, \sigma)=e^{-\frac{1}{2} b^{2} \lambda^{2}} e^{-i c_{\sigma} \lambda^{2}}\left(\sum_{j=0}^{N} \lambda^{j} A_{j}\right)
$$

where $c_{\sigma}$ are real constants.

We now indicate how to prove Theorem 1.5. The first thing to do is to show that the function $F(\lambda)=(\hat{f}(\lambda, \sigma) \varphi, \psi)$ extends to $\mathfrak{a}_{\mathbb{C}}^{*}$ as an entire function of order 2. Once this is done we can proceed as in Theorem 1.4 to show that

$$
\hat{f}(\lambda, \sigma)=e^{-\left(E_{\sigma} \lambda, \lambda\right)}\left(\sum_{|\alpha| \leq N} \lambda^{\alpha} A_{\alpha}\right) .
$$

It is clear that $R e E_{\sigma}$ has to be positive definite. We now proceed to estimate $F(\lambda)$.

As we are assuming that $f(x) e^{a|x|^{2}}$ belongs to $L^{p}(G)$ it is easy to estimate $F(\lambda)$. In fact, as $F(\lambda)=(\hat{f}(\lambda, \sigma) \varphi, \psi)$

$$
|F(\lambda)| \leq c\|\varphi\|_{2}\|\psi\|_{2} \int_{G}|f(x)| e^{|\operatorname{Im}(\lambda)||x|} d x .
$$

Applying Hölder's inequality the above integral is bounded by the product of

$$
\int_{G}|f(x)|^{p} e^{a p|x|^{2}} d x
$$

and

$$
\begin{aligned}
& \int_{G} e^{-a q|x|^{2}} e^{|\operatorname{Im}(\lambda)||x|} d x \\
\leq & c \int_{A} e^{-a q|\log a|^{2}} e^{|\operatorname{Im}(\lambda)||\log a|} e^{2 \rho(\log a)} d a \\
\leq & c e^{\beta|\operatorname{Im}(\lambda)|^{2}} .
\end{aligned}
$$

This proves that $F(\lambda)$ is entire of order 2 .

Now we have to show that the entire function

$$
\int_{0}^{z \xi_{1}} \ldots \int_{0}^{z \xi_{\ell}} F(w) \overline{F\left(e^{i \alpha} \bar{w}\right)} d w
$$

has polynomial growth. Again this is easily done. It is enough to consider

$$
\int_{|\lambda| \leq R} F(\lambda) F(i \lambda) d \lambda
$$


as the estimation of other integrals are similar. First consider the integral

$$
\begin{aligned}
& \int_{|\lambda| \leq R} \int_{|x| \leq \frac{2}{a} R}|F(\lambda)||f(x)| e^{|\lambda||x|} d x d \lambda \\
\leq & c(1+R)^{2 N} \int_{\mathfrak{a}^{*}} \int_{G} \frac{|F(\lambda)|}{(1+|\lambda|)^{N}} \frac{|f(x)|}{(1+|x|)^{N}} e^{|\lambda||x|} d x d \lambda \\
\leq & c\|\varphi\|_{2}\|\psi\|_{2}(1+R)^{2 N} .
\end{aligned}
$$

The remaining part of the integral is

$$
\begin{aligned}
& \int_{|\lambda| \leq R} \int_{|x|>\frac{2 R}{a}}|F(\lambda)||f(x)| e^{|\lambda||x|} d x d \lambda \\
\leq & \left(\int_{|\lambda| \leq R}|F(\lambda)| d \lambda\right)\left(\int_{|x|>\frac{2 R}{a}}|f(x)| e^{R|x|} d x\right) .
\end{aligned}
$$

The second integral on the right hand side is bounded by

$$
\left(\int_{G}|f(x)|^{p} e^{a p|x|^{2}} d x\right)^{\frac{1}{p}}\left(\int_{|x|>\frac{2 R}{a}} e^{-a q|x|^{2}+R q|x|} d x\right)^{\frac{1}{q}}
$$

where $\frac{1}{p}+\frac{1}{q}=1$. Since $|x|>\frac{2 R}{a}$ the second integral is bounded by

$$
\int_{G} e^{-\frac{q}{2} q|x|^{2}} d x \leq c
$$

Hence we are in a position to proceed as before to arrive at the conclusion of Theorem 1.5.

\section{References}

[1] Anker, J.-P., Sharp estimates for some functions of the Laplacian on noncompact symmetric spaces, Duke J. Math., 65 (1992), 257-297.

[2] Anker, J-P., Damek, E. and Yacoub, C., Spherical analysis on harmonic $A N$ groups, Ann. Scoula Norm. Sup. Pisa, 23 (1996), 643-679.

[3] Anker, J-P. and Ji, L., Heat kernel and Green function estimates for noncompact symmetric spaces, Geom. Funct. Anal., 9 (1999), 1035-1091.

[4] Astengo, F., Cowling, M., Di Blasio, B. and Sundari, M., Hardy's uncertainty principle on some Lie groups, J. London Math. Soc., 62 (2000), 461-472.

[5] Bagchi, S. C. and Ray, S., Uncertainty principles like Hardy's theorem on some Lie groups, J. Aust. Math. Soc., 65 (1998), 289-302.

[6] Bonami, A., Demange, B. and Jaming, P., New uncertainty principles for the Fourier and windowed Fourier transform, Rev. Math. Ibero., 19 (2003), 23-55.

[7] Bray, W., Generalised spectral projections on symmetric spaces of non-compact type; Paley-Wiener theorems, J. Funct. Anal., 135 (1996), 206-232. 
[8] Cowling, M. and Price, J., Generalisations of Heisenberg's inequality, in Harmonic Analysis (G. Mauceri, F. Ricci and G. Weiss, eds), Lecture Notes in Math., 992, Springer, Berlin (1983).

[9] Cowling, M., Sitaram, A. and Sundari, M., Hardy's uncertainty principles on semisimple groups, Pacific J. Math., 192 (2000), 293-296.

[10] Eguchi, M., Koizumi, S. and Kumahara, N., An $L^{p}$ version of the Hardy theorem for motion groups, J. Aust. Math. Soc. Ser. A, 68 (2000), 55-67.

[11] _ An analogue of the Hardy theorem for the Cartan motion groups, Proc. Japan Acad., 74 (1998), 149-151.

[12] Folland, G. B. and Sitaram, A., The uncertainty principle: A mathematical survey, J. Fourier Anal. Appl., 3 (1997), 207-238.

[13] Gelfand, I. M. and Shilov, G. E., Fourier transforms of rapidly increasing functions and questions of uniqueness of the solution of Cauchy's problem, Uspekhi Mat. Nauk., 8 (1953), 3-54.

[14] Gross, K. I. and Kunze, R. A., Fourier decompositions of certain representations, in symmetric spaces, Boothby and Weiss eds, Marcel-Decker, New York, (1972), 119-139.

[15] Gangolli, R. and Varadarajan, V. S., Harmonic Analysis of Spherical functions, Springer-Verlag, 1988.

[16] Hardy, G. H., A theorem concerning Fourier transforms, J. London Math. Soc., 8 (1933), 227-231.

[17] Hörmander, L., A uniqueness theorem of Beurling for Fourier transform pairs, Ark. Mat., 29 (1991), 237-240.

[18] Johnson, K. D. and Wallach, N., Composition series and intertwining operators for the spherical principal series I, Trans. Amer. Math. Soc., 229 (1977), 137-174.

[19] Kaniuth, E. and Kumar, A., Hardy's theorem for simply connected nilpotent Lie groups, Math. Proc. Camb. Phil. Soc., 131 (2001), 487-494.

[20] Knapp, A., Representation theory of semisimple groups, Princeton Univ. Press, Princeton, 1986.

[21] Koornwinder, T. H., The representation theory of $S L(2, \mathbb{R})$, a non infinitesimal approach, Enseign. Math., 28 (1982), 53-90.

[22] _ Jacobi functions and analysis on noncompact semisimple Lie groups, in Special functions: Group theoretical aspects and applications, R. Askey, T. H. Koornwinder and W. Schempp eds., Reidel Dordrecht, 1984.

[23] Narayanan, E. K. and Ray, S. K., $L^{p}$ version of Hardy's theorem for semisimple Lie groups, Proc. Amer. Math. Soc., 130 (2002), 1859-1866.

[24] Proc. Ind. Acad. Sci., 112 (2002), 321-330.

[25] Pfannschmidt, C., A generalisation of the theorem of Hardy: A most general version of the uncertainty principle for Fourier integrals, Math. Nachr., 182 (1996), 317-327.

[26] Ray, S. K., Uncertainty principles on two step nilpotent Lie groups, Proc. Ind. Acad. Sci., 111 (2001), 1-26.

[27] Ray, S. K. and Sarkar, R., $L^{p}$ version of Hardy's theorem and characterisation of heat kernels on symmetric spaces, Preprint, 2001.

[28] Sarkar, R., A complete analogue of Hardy's theorem on semisimple Lie groups, Colloq. Math., 93 (2002), 27-40.

[29] Sarkar, R., A complete analogue of Hardy's theorem on $S L_{2}(\mathbb{R})$ and characterisation of the heat kernel, Proc. Ind. Acad. Sci., 112 (2002), 579-593.

[30] Sarkar, R. and Thangavelu, S., On theorems of Beurling and Hardy for the Euclidean motion group, Preprint, 2002.

[31] Sengupta, J., An analogue of Hardy's theorem for semisimple Lie groups, Proc. Amer. Math. Soc., 128 (2000), 2493-2499.

[32] - The uncertainty principle on Riemannian symmetric spaces of the noncompact type, Proc. Amer. Math. Soc., 130 (2002), 1009-1017. 
[33] Shimeno, N., An analogue of Hardy's theorem for the Harish-Chandra transform, Hiroshima Math. J., 31 (2001), 383-390.

[34] Sitaram, A., Sundari, M. and Thangavelu, S., Uncertainty principles on certain Lie groups, Proc. Ind. Acad. Sci., 105 (1995), 135-151.

[35] Sitaram, A. and Sundari, M., An analogue of Hardy's theorem for very rapidly decreasing functions on semisimple groups, Pacific J. Math., 177 (1997), 187-200.

[36] Sundari, M., Hardy's theorem for the $n$-dimensional Euclidean motion group, Proc. Amer. Math. Soc., 126 (1998), 1199-1204.

[37] Thangavelu, S., An analogue of Hardy's theorem for the Heisenberg group, Colloq. Math., 87 (2001), 137-145.

[38] _ Revisiting Hardy's theorem for the Heisenberg group, Math. Z., 242 (2002), $761-779$

[39] Hardy's theorem for the Helgason Fourier transform on rank one symmetric spaces, Colloq. Math., 94 (2002), 263-280.

[40] Varadarajan, V. S., Lie groups Lie algebras and their representations, Springer-Verlag, 1984. 